\title{
TOTALLY CATEGORICAL STRUCTURES
}

\author{
EHUD HRUSHOVSKI
}

\begin{abstract}
A first order theory is totally categorical if it has exactly one model in each infinite power. We prove here that every such theory admits a finite language, and is finitely axiomatizable in that language, modulo axioms stating that the structure is infinite. This was conjectured by Vaught. We also show that every $\aleph_{0}$-stable, $\aleph_{0}$-categorical structure is a reduct of one that has finitely many models in small uncountable powers. In the case of structures of disintegrated type we nearly find an explicit structure theorem, and show that the remaining obstacle resides in certain nilpotent automorphism groups.
\end{abstract}

\section{INTRODUCTION}

A sequence of fundamental papers in model theory has given rise to an understanding of the totally categorical structures as those structures that are coordinatized (in a certain precise way) by a projective space over a finite field (or in the degenerate case by a pure set). Much of this work was motivated by a conjecture of Vaught: a totally categorical theory can be axiomatized by a finite set of sentences that has arbitrarily large finite models, together with axioms stating that the intended models are infinite. The part stating that every finite set of axioms does have a finite model was settled in [Z] and refined in [CHL]. $\S 2$ of the present paper proves the conjecture in full. In particular, it follows that the class of totally categorical theories is countable.

In [CHL], the context of research was shifted from totally categorical theories to $\aleph_{0}$-categorical, $\aleph_{0}$-stable ones; those theories whose models are coordinatized by a collection of projective spaces over finite fields and degenerate spaces (rather than just one). Lachlan proved that in the disintegrated case, where only degenerate spaces occur, these structures are precisely the reducts of the totally categorical ones [L]. In $\S 3$ we prove the corresponding result in general: the $\aleph_{0}$-stable, $\aleph_{0}$-categorical structures are precisely the reducts of $\aleph_{0}$-categorical structures whose theories have finitely many models in some uncountable power. The number of dimensions of the expanded structure will be the number of distinct primes occurring as characteristics of finite fields associated with the projective spaces in the original structures. The main point of the proof is a trick needed to negate the effect of finite Galois groups.

Received by the editors April 13, 1987.

1980 Mathematics Subject Classification (1985 Revision). Primary 03C45.

Key words and phrases. Categoricity, stability, finite axiomatizability, coordinatization by projective space, strongly minimal sets. 
The theorem of $\S 1$ implies that there are only countably many $\aleph_{0}$-categorical, $\aleph_{0}$-stable theories, but gives no explicit structure theorem. In $\S 4$ we attempt to give one, assuming that the coordinatizing strongly minimal set is disintegrated. (Most of the results require only the weaker assumption that no affine spaces are present.) We give an explicit construction of a certain class of disintegrated totally categorical structures. It falls short of containing all of them, but not by much: every disintegrated totally categorical structure expands to a member of the class by simply naming a finite set of parameters. (In particular, we obtain a direct proof of the original Ahlbrandt-Ziegler result in this case.) We also show that the information lost in the naming of parameters is controlled by nilpotent automorphism groups. As a corollary, the automorphism group of such a structure has a finite Jordan-Hölder decomposition (as a topological group) in which each of the components are of a known type.

Aside from the basic results that set the groundwork (Morley, BaldwinLachlan, Zilber, Cherlin-Harrington-Lachlan), this paper has several direct precursors. The basic idea of $\S 2$ originated with [A-Z], where the conjecture was proved in the almost strongly minimal case. Lachlan then proved the finite language result for structures of disintegrated type. The common generalization was then achieved by Cherlin, who proved both finite language and quasi-finite axiomatizability for the class of $\aleph_{0}$-categorical, $\aleph_{0}$-stable structures of modular type. (One needs to redefine quasi-finite axiomatizability in this context to take into account the existence of several dimensions.) The basic setup for the general proof was already present there.

$\S 3$ generalizes a result from [L]. $\S 4$ can also be viewed as strengthening [L], for example the result there that every totally categorical structure of disintegrated type is interpretable in a dense linear ordering. It seems clear that every $\aleph_{0}$-categorical, $\aleph_{0}$-stable structure of modular type is interpretable in a finite disjoint union of universal locally lexicographically ordered vector spaces over finite fields; see [T]. This will not be pursued.

The notation is from [CHL], and the basic facts proved or assumed there are assumed here also. $\left(d_{p} x\right) \varphi(x, y)$ denotes the $p$-definition of $\varphi$.

Greg Cherlin helped with this paper in many ways other than the obvious debt to his [C]; I would like to thank him.

\section{Finite language AND aXiomatizability}

Theorem 2.1. (a) Let $M$ be $\aleph_{0}$-categorical, $\aleph_{0}$-stable. Then $M$ admits a finite language.

(b) If a finite language is chosen for $M$, then the theory of $M$ is quasi-finitely axiomatizable.

A theory $T$ is quasi-finitely axiomatizable iff there exist a finite $T_{0} \subset T$ and a finite number of axiom schemes of infinity true in models of $T$, which together axiomatize $T$. An axiom scheme of infinity is a collection of axioms that assert for certain formulas $\varphi$ and $D$ that in any model of $T$, if $\models \varphi(\bar{b})$ 
then $D_{\bar{b}}$ is infinite in $M$. (Here $D=D(x, \bar{y}), D_{\bar{b}}=\{x: \vDash D(x, \bar{b})\}$, and imaginary sorts are allowed.)

Remark 2.2. One an restrict the allowable sorts in various ways without changing the content of the definition. However, unless certain axiom schemes of homogeneity are added, it is not in general enough to have axioms stating that each class in one 0 -definable equivalence relation is split into infinitely many classes by a second one.

If $T$ is totally categorical, it is easy to see that the only axiom scheme of infinity needed is the one stating that the model is infinite.

The theorem will be proved by the Ahlbrandt-Ziegler method. It also owes a large debt to Cherlin's [C]. The fine structure of $\aleph_{0}$-categorical, $\aleph_{0}$-stable models is hardly used at all. Indeed, it seems that no truly essential use is made of our knowledge of the identity of the strictly minimal sets involved, except for the fact that they admit a quasi-well-ordering.

After replacing $M$ by a bi-interpretable model, we will define a partially ordered set $\Pi$ and an order preserving map $U: p \mapsto U_{p}$ of $(\Pi,<)$ into subsets of $M$, satisfying the following conditions:

(C0) (Normality) The action of $\operatorname{Aut}(M)$ on $M$ extends to an action on $(\Pi,<, U, M)$.

(C1) ( $\Pi$ is ample) (a) $\Pi$ has a least element, whose image is $\varnothing$, and a greatest, with image $M$.

(b) If $p<q$ then there exists $p^{\prime}$ with $p<p^{\prime} \leq q$ such that $U_{p^{\prime}}=U_{p} \cup\{a\}$ for a singleton $a$. Moreover, one may choose $\operatorname{tp}\left(a / U_{p}\right)$ to be either algebraic or strongly minimal.

(c) Any increasing sequence $p_{1} \leq p_{2} \leq \cdots$ in $\Pi$ has a least upper bound $p \in \Pi$; and $U_{p}$ is the union of the $U_{p_{i}}$ 's.

(C2) (Quasi-well-foundedness) Let $\mathscr{W}$ be the range of $U$. For any finite $F \subset M$ and any $S_{1}, S_{2}, \ldots \in \mathscr{W}$ there exist $i<j<\omega$ and an isomorphism $h$ of $M$ such that $h$ fixes $F$ pointwise and $h\left(S_{i}\right) \subset S_{j}$.

(C3) (Uniform finite language property) There exists a finite set $L$ of 0 definable relations such that for any $S \in \mathscr{W}$, any map $h: S \rightarrow M$ preserving the relations in $L$ is a partial elementary map.

(C4) Every $S \in \mathscr{W}$ is a definable set.

The "moreover" clause of $(\mathrm{Cl})(\mathrm{b})$ creates a somewhat artificial distinction between different Morley ranks. The only reason for this discrimination lies in the definition of quasi-finite-axiomatizability, which says something about formulas of rank 1 only, and thus creates an asymmetry. We will not need this clause until the very end. (C4) is not necessary for the finite language result, and is too weak for the finite axiomatizability (we will need a sharper version); but it seems reasonable to include it. (C3) will immediately be seen to be redundant.

Lemma 2.3. (C0)-(C2) imply (C3) .

To prove this we need a "downward Lowenheim-Skolem" principle. 
Lemma 2.4. For each integer $k$ there exists an integer $\lambda=\lambda(k)$ such that for all $S \in \mathscr{W}$ and all $k$-element subsets $F$ of $M$, there exists a subset $H$ of $S$ of cardinality at most $\lambda$ such that:

$$
\begin{aligned}
& *(F, S, H): F \cap S \subseteq H, F, S \text { are independent over } H \text { and } \\
& \operatorname{tp}(F / S) \text { is the unique nonforking extension to } S \text { of } \operatorname{tp}(F / H) .
\end{aligned}
$$

Proof. Fix $F$. For $S \in \mathscr{W}$, let $\lambda(F, S)$ be the least possible cardinality of a subset $H$ such that $*(F, S, H)$. Suppose $\lambda(F, S)$ can be unboundedly large. By Ramsey's theorem, one can then find a chain $S_{m}(m \in \omega)$ of elements of $\mathscr{W}$ such that $\lambda\left(F, S_{m}\right) \geq m$, and either each $S_{i}$ embeds into $S_{i+1}$ by a partial elementary map fixing $F$, or no $S_{i}$ embeds into any $S_{j}(j>i)$ in this way. The second possibility contradicts $(\mathrm{C} 2)$ directly. So replacing each $S_{i}$ in turn by a conjugate copy over $F$, we may assume that the $S_{j}$ 's form an increasing chain, with union $S$ (say). By $\omega$-stability it follows that for some $i_{0}, *\left(F, S_{i_{0}}, S\right)$ holds. Therefore if $*\left(F, H, S_{i_{0}}\right)$ then $*\left(F, H, S_{i}\right)$ holds for all $i \geq i_{0}$. Hence $\lambda\left(F, S_{i_{0}}\right) \geq \lambda\left(F, S_{i}\right)$ for each $i$, a contradiction. Thus $\lambda(F, S)$ is bounded by some $\lambda(F)$. Since $\lambda(F)$ depends only on $\operatorname{tp}(F)$ and there are only finitely many possibilities for $\operatorname{tp}(F), \lambda$ can be chosen to depend on $k$ alone.

Let $\lambda=\lambda(1)$, and let $L$ be a finite set of relations containing a logical equivalent to every $k$-ary relation for $k \leq \lambda+1$. I claim that this $L$ satisfies (C3). We will show that if $\mathbb{C}$ is a large, saturated elementary extension of $M$, $S=U_{p}$, and $h: S \rightarrow \mathbb{C}$ preserves the relations in $L$, then $h$ is elementary. By $(\mathrm{Cl})(\mathrm{c})$ and Zorn's lemma, one can find a $\Pi$-maximal $q \in \Pi$ such that $q \leq p$ and $h \mid U(q)$ is elementary. Let $T=U(q) . h \mid T$ extends to an automorphism of $\mathbb{C}$. Without loss of generality, $h$ is the identity on $T$. Suppose for contradiction that $T \neq S$. By $(\mathrm{C} 1)(\mathrm{b})$ there exists $a \in S-T$ such that $T \cup\{a\} \in \mathscr{W}$. Replacing $M, S, T$ by $h(M), h(S), h(T)$ and $h$ by $h^{-1}$ if necessary, we may assume that $\operatorname{rk}(a / T) \leq \operatorname{rk}(h(a) / T)$. By Lemma 2.4, there exists $F \subset T$ with $\operatorname{card}(F) \leq \lambda$ such that $\operatorname{tp}(a / T)$ is the unique nonforking extension of $\operatorname{tp}(a / F)$. As $h$ preserves $L, \operatorname{tp}(h(a) / F)=\operatorname{tp}(a / F)$. Because of the rank inequality, $h(a)$ must realize the unique nonforking extension $T$ of $\operatorname{tp}(a / F)$. Thus $h \mid T \cup\{a\}$ is elementary, contradicting the maximality of $T$. Thus $T=S$, so $h$ is elementary.

In particular, this proves that part (a) of the theorem holds, assuming the existence of a good collection $\mathscr{W}$. To prove part (b), and to find $\Pi, U, \mathscr{W}$, we need to change $M$ slightly. By Lemma 3.8 we may assume momentarily that $M$ has a unique 1-type. Let $c$ realize this type. By repeated applications of the coordinatization theorem of [CHL], there exists a sequence $a_{1}, \ldots, a_{n}$ such that $C \in \operatorname{dcl}\left(a_{n}\right)$ and $\operatorname{tp}\left(a_{i+1} /\left\{a_{1}, \ldots, a_{i}\right\}\right)$ is strictly minimal or algebraic for each $i$. Replacing $a_{i+1}$ by $\left(a_{1}, \ldots, a_{i}, a_{i+1}\right)$, we may assume that $a_{i} \in \operatorname{dcl}\left(a_{i+1}\right)$ for each $i$. Let $r_{i}=\operatorname{tp}\left(a_{1}, \ldots, a_{i}\right)$, and let $L\left(a_{1}, \ldots, a_{i-1}\right)=\left\{\left(x_{1}, \ldots, x_{i}\right) \models\right.$ $r_{i}: x_{j}=a_{j}$ for $\left.j>i\right\}$. There are four possibilities for $L\left(a_{1}, \ldots, a_{i-1}\right)$ : it 
may be modular and orthogonal to each $L\left(a_{1}, \ldots, a_{j-1}\right)$ for $j<i$, it may be modular but nonorthogonal to some previous $L\left(a_{1}, \ldots, a_{j-1}\right)$; it may be affine; or it may be finite. The cases do not depend on the choice of $\bar{a} \models r$, so they give a partition of $\{1, \ldots, n\}$ into four sets, which we will call $I_{\text {new }}, I_{\text {old }}, I_{\text {afn }}$ and $I_{\text {fin }}$, respectively. We make the following further requirements. If $i \in I_{\text {afn }}$ then the corresponding projective space should already have been encountered as $L\left(a_{1}, \ldots, a_{j-1}\right)$ for some $j<i$. If $L\left(a_{1}, \ldots, a_{i}\right)=P$ is a projective space over a field $F$, then $F$ can be considered as a (finite) definable subset of $M$; an element of $F$ can be taken to be a certain equivalence class of 4-tuples from $P$. We require that each element of $F$ be definable over $a_{1}, \ldots, a_{i-1}$. The effect of this is that for any tuple $\bar{x}$ from $P, \operatorname{tp}\left(\bar{x} / a_{1} \cdots a_{i-1}\right)$ is stationary. For $i \in I_{\text {new }} \cup I_{\text {old }} \cup I_{\text {afn }}$, let $\alpha(i)$ be the least $j \leq i$ such that $L\left(a_{1}, \ldots, a_{i-1}\right)$ is nonorthogonal to $L\left(a_{1}, \ldots, a_{j-1}\right)$ (if $\bar{a} \models r_{n}$ ).

We will work not in $M$ but in $M^{*}$, the $n$-sorted model whose sorts are the solution sets to $r_{1}, \ldots, r_{n}$. As $M$ and $M^{*}$ are bi-interpretable, the transition can be made. $\aleph_{0}$-stability, $\aleph_{0}$-categoricity, and quasi-finite axiomatizability are invariant under this transformation, the last essentially by definition. $M^{*}$ has a natural tree structure: a node of height $k$ is a realization of $r_{k}$. The branches correspond to realizations $\left(a_{1}, \ldots, a_{n}\right)$ of $r_{n}$, ordered by $a_{1}<a_{1}<\cdots<a_{n}$. We will sometimes think of the tree as having a root, a formal element which will always be denoted $a_{0}$. From now on we assume that $M=M^{*}$, i.e. that one has types $r_{i}$ and a tree structure satisfying the above requirements. Having moved from $M$ to $M^{*}$, we will not make further use of imaginary elements.

Property (C2) will ultimately come from the following lemma, which in turn is a translation of a combinatorial lemma of Higman's. It is essentially equivalent to the existence of a good enumeration of a projective space, proved in [A-Z], but does not appear to follow directly from any statement there. The proof will be given at the end of the section.

Lemma 2.5. Let $P$ be a projective space over a finite field. Then there exists an ordering of $P$ of order type $\omega$ with the following property. Let $\bar{x}_{p}=\left(x_{p}^{1}, \ldots, x_{p}^{n}\right)$ be an $n$-tuple of elements of $P$ for $p=1,2, \ldots$. Then for some $p_{0}<p_{1}$ there exists an order preserving elementary embedding $h: P \rightarrow P$ with $h\left(\bar{x}_{p_{0}}\right)=\left(\bar{x}_{p_{1}}\right)$.

The same lemma holds trivially for a disintegrated strongly minimal set, taking any ordering of order type $\omega$. To avoid mentioning this case explicitly we will consider such sets to be degenerate projective spaces, over a "1-element field".

For each finite field $F$, fix a countable-dimensional projective space $(P,<)$ over $F$, ordered as in the lemma. An ordering isomorphic to $(P,<)$ will be called standard. Given such an ordering on $P$, let $P^{3}$ be ordered as follows:

$$
(a, b, c)<\left(a^{\prime}, b^{\prime}, c^{\prime}\right) \quad \text { iff } \quad(\max (a, b, c), a, b, c)
$$


precedes $\left(\max \left(a^{\prime}, b^{\prime}, c^{\prime}\right), a^{\prime}, b^{\prime}, c^{\prime}\right)$ lexicographically. So $P^{3}$ also has ordertype $\omega$, and every order-preserving embedding $h: P \rightarrow P$ induces an orderpreserving map $P^{3} \rightarrow P^{3}$.

Claim 2.6. (a) Let $i \in I_{\text {afn }}$. Then there exists a $\left(q_{i}+3\right)$-place 0-definable map $f_{i}$ with the following property. Given $\left(a_{1}, \ldots, a_{i}\right) \vDash r_{i}$, let $P=$ $L\left(a_{1}, \ldots, a_{\alpha(i)-1}\right), L=L\left(a_{1}, \ldots, a_{i-1}\right)$. Let $l$ be an enumerated affine line in $L$. Then the map $\pi_{1}: P^{3} \rightarrow L$ defined by: $\pi(\bar{x})=f_{i}(l, \bar{x})$ is well defined and surjective.

(b) Let $i \in I_{\text {old }}$. Then there exsits an $(i-1)+1$-place 0 -definable map $f_{i}$ such that for any $\bar{a}=\left(a_{1}, \ldots, a_{i-1}\right) \vDash r_{i-1}$, if $j=\alpha(i), P=L\left(a_{1}, \ldots, a_{\alpha(i)-1}\right)$, and $L=L\left(a_{1}, \ldots, a_{i-1}\right)$, then the map $\pi_{\bar{a}}: P \rightarrow L$ defined by $\pi(x)=f_{i}(\bar{a}, x)$ is surjective.

Proof. (b) is obvious. To prove (a), let $a_{1}, \ldots, a_{i}$ and $l$ be given, and let $b^{0}, b^{1}$ be the first two points of $l$. Let $\hat{L}$ be the projective space of directions in $L$. For any $x \in L-l$, let $\alpha^{i}(x)$ be the direction from $b^{i}$ to $x$, and let $\alpha(x)=\left(\alpha^{0}(x), \alpha^{1}(x)\right)$. Clearly $\alpha$ is a $1-1$ map of $L-l$ into $\hat{L}^{2}$. So $\alpha^{-1}$ is a map defined on a certain subset of $\hat{L}^{2}$, onto $L-l$. It is easy to doctor $\alpha^{-1}$ so as to get a map of $\hat{L}^{3}$ onto $L$. Composing with the projection $\pi: P \rightarrow \hat{L}$ obtained as in (b), we get a map $P^{3} \rightarrow L$. The process was uniform in $\bar{a}, l$.

Definition 2.7. We will first define a collection $\mathscr{W}_{0}$ of subsets of $M$. Each $U \in$ $\mathscr{W}_{0}$ will have a certain characteristic subset of $U$ called the base. The base will have the form $S_{0} \cup\left\{a_{0}\right\} \cup S_{1} \cup\left\{a_{1}\right\} \cup \cdots \cup S_{k}$ for certain sets $S_{i} \subset\left\{x \in U: x \models r_{i}\right\}$ and certain distinguished elements $a_{i}$. Let $\leq$ denote the tree ordering of $M$, described previously. If $U=M$ then $S_{0}=\left\{a_{0}\right\}$ and $k=0$. Otherwise, $S_{0}=\varnothing$, and $a_{0}$ is the root of the tree. Assume $S_{j}$ and $a_{j}$ have been defined for $j<i$. Let $S_{i}=\left\{x \models r_{i}: a_{i-1} \leq x\right.$, and whenever $\left.x \leq y \in M, y \in U\right\}$. In order that $U$ be in $\mathscr{W}_{0}$, we require that either $U \cap\left\{x: x \geq a_{i-1}\right\}$ is precisely the upward closure of $S_{i}$ in the tree, or else there exists a unique element $a_{i} \vDash r_{i}$, $a_{i} \geq a_{i-1}$, such that every element of $U$ above $a_{i-1}$ lies above an element of $S_{i} \cup\left\{a_{i}\right\}$. The definition of the base stops when the first case occurs. The base has actually been defined as a structured set $\left(a_{0}, S_{1}, a_{1}, \ldots, S_{k}, a_{k}\right)$, and we will treat it as such.

Definition 2.8. We now define $\Pi, U$ and $\mathscr{W} . \Pi$ is the set of all objects of the form $p=\left(S_{1}, a_{1}, \ldots, S_{k} ; T_{1}, \ldots, T_{k} ;<_{1}, \ldots,<_{k} ; l_{1}, \ldots, l_{k}\right)$, satisfying the following. $\left(S_{1}, a_{1}, \ldots, S_{k}\right)$ are the base of some set $U \in \mathscr{W}_{0}$. Clearly $U$ is uniquely determined; by definition this is $U(p) .<_{i}$ occurs in $p$ only if $i \in I_{\text {new }}$; in that case it is a standard ordering of $L\left(a_{1}, \ldots, a_{i-1}\right)$. If $i \in$ $I_{\text {new }} \cup I_{\text {old }}, T_{i}$ is a subset of $L\left(a_{1}, \ldots, a_{\alpha(i)-1}\right)$; if $i \in I_{\text {afn }}, T_{i}$ is a subset of $L\left(a_{1}, \ldots, a_{\alpha(i)-1}\right)^{3}$. If $i \in I_{\text {new }} \cup I_{\text {fin }}, T_{i}=S_{i} . l_{i}$ occurs iff $i \in I_{\text {afn }}$, and then it is an ordered affine line from $L\left(a_{1}, \ldots, a_{i-1}\right)$. The main conditions: for $i \in$ $I_{\text {new }} \cup I_{\text {old }} \cup I_{\text {afn }}$, each $T_{i}$ is an initial section of the ordering on the appropriate 
set, and $S_{i} \cup\left\{a_{i}\right\}$ is the image of $T_{i}$ under $f_{i}(l,-)$ or $f_{i}\left(a_{1}, \ldots, a_{i-1,-}\right)$. This defines $\Pi$ and $\mathscr{W}$ as sets. The ordering on $\Pi$ is defined as follows. $p<p^{\prime}$ if $U_{p} \subset U_{p^{\prime}}$, and for each $j$, if $a_{j}=a_{j}^{\prime}$ then $<_{j+1}=<_{j+1}^{\prime}$ or $l_{j+1}=l_{j+1}^{\prime}$ (at most one of these conditions can apply).

We proceed to prove that $(\Pi, U, \mathscr{W})$ satisfy conditions $(\mathrm{C} 0)-(\mathrm{C} 4)$. (C0) is obvious. So is $(\mathrm{C} 4)$, but we record a more precise version.

$\left(C 4^{\#}\right)$ Given $U \in \mathscr{W}$ one can canonically define a finite set $B=\operatorname{base}(U) \subseteq$ $U$. $B$ decomposes canonically into a "bounded part" $\bar{a}_{U}$ and a "monotone part" $T$. This means that $\operatorname{base}(U)=\left\{a_{0}, \ldots, a_{i}\right\}_{U} \cup T_{U}$, where $i$ is bounded independently of $U$, and if $\bar{a}_{U}=\bar{a}_{U^{\prime}}$, then $U \subset U^{\prime}$ implies $T_{U} \subset T_{U^{\prime}}$. Further, $U$ is definable from $B$ : there exists a formula $\varphi(x, y)$ such that $U=\left\{x: \varphi(x, y)\right.$ holds for some $\left.y \in T_{U}\right\} \cup\left\{a_{0}, \ldots, a_{i}\right\}$.

That $\left(\mathrm{C}^{\#}\right)$ holds in $M$ is clear: letting base $(U)=S_{0} \cup\left\{a_{0}\right\} \cup \cdots \cup S_{k}$, the bounded part is $\left\{a_{0}, \ldots, a_{k-1}\right\}$ and the monotone part is $S_{0} \cup S_{1} \cup \cdots \cup S_{k}$. $\varphi(x, y)$ says that $x$ is above $y$ in the tree ordering.

Lemma 2.9. ( $\Pi, U)$ satisfies $(\mathrm{C} 1)$.

Proof. $(\mathrm{C} 1)(\mathrm{a})$ and $(\mathrm{b})$ are easy. To prove $(\mathrm{Cl})(\mathrm{c})$, let $p^{1}<p^{2}<\cdots$ in $\Pi$. As a matter of notation, let superscripts and brackets commute in expressions such as

$$
p^{j}=\left(S_{0}, a_{0}, \ldots, S_{k} ; T_{1}, \ldots, T_{k} ;<_{1}, \ldots,<_{k} ; l_{1}, \ldots, l_{k}\right)^{j} .
$$

Let

$$
p^{j} \mid i=\left(S_{0}, a_{0}, \ldots, S_{i} ; T_{1}, \ldots, T_{i} ;<_{1}, \ldots,<_{i} ; l_{1}, \ldots, l_{i}\right)^{j},
$$

and let $i$ be the largest integer $\leq k$ such that the sequence $\left(p^{j} \mid i\right)$ is eventually constant. Call its eventual value $(p \mid i)^{\infty}$. If $i=k$ there is nothing to prove, so assume $i<k$. Note that $a_{i}^{j}$ must also have a constant value $a_{i}^{\infty}$ eventually: if $p\left|i=p^{\prime}\right| i$ but $a_{i}^{p} \neq a_{i}^{p^{\prime}}$ then $p, p^{\prime}$ are incomparable in $\Pi$. Our choice for the limit of the $p^{j}$ 's is

$$
\begin{aligned}
p=\left(S_{0}^{\infty}, a_{0}^{\infty}, S_{1}^{\infty}, \ldots, a_{i-1}^{\infty}, S_{i}^{\infty} \cup\left\{a_{i}^{\infty}\right\} ; T_{1}^{\infty}\right. & , \ldots, T_{i}^{\infty} ; \\
& \left.<_{1}^{\infty}, \ldots,<_{i}^{\infty} ; l_{1}^{\infty}, \ldots, l_{i}^{\infty}\right) .
\end{aligned}
$$

It follows from the definition of the base of a set that $S_{i+1}^{j} \subseteq S_{i+1}^{j+1} \subseteq \cdots$ for large enough $j$. We need to distinguish three cases.

If $i+1 \in I_{\text {fin }}$. Since $T_{i+1}=S_{i+1}$ and $<_{i+1}, l_{i+1}$ are undefined for $i+1 \in I_{\text {fin }}$, it follows that $\left(p^{j} \mid i+1\right)$ converges also, contradicting the maximality of $i$.

If $i+1 \in I_{\text {new }}$. By the definition of the ordering on $P,<_{i+1}^{j}$ is eventually constant (as soon as $a_{i}^{j}$ is). So $S_{i+1}^{j}$ is an initial section of $<_{i+1}^{\infty}$. Since this ordering has order type $\omega$, there are two cases: either the value of $S_{i+1}^{j}$ stabilizes eventually, or else the union of the $S_{i+1}^{j}$ 's is $L\left(a_{1}^{\infty}, \ldots, a_{i}^{\infty}\right)$. In the first case, $p^{j} \mid i+1$ stabilizes, a contradiction. In the second, a straightforward check shows that $(p \mid i)^{\infty}$ satisfies the requirements. 
If $i+1 \in I_{\mathrm{afn}}$ or $I_{\mathrm{old}}$. Then $\alpha(i+1) \leq i$, so the relevant ordering $<_{\alpha(i+1)}^{j}$ stabilizes. By the definition of the ordering on $\Pi, l_{i+1}^{j}$ also has a constant value eventually in the affine case. Thus for large $j, S_{i+1}^{j}$ is the image of $T_{i+1}^{j}$ under a fixed function. Since $T_{i+1}^{j}$ is an initial section of $<_{\alpha(i+1)}^{\infty}$ for large $j$, the same argument as before works.

Lemma 2.10. (C2) holds.

Proof. Define partial orderings " $\leq W$ (up to $k$ )" on $\Pi \times M^{r}$ as follows. $(p, \bar{c})$ $\leq^{W}\left(p^{\prime}, \overline{c^{\prime}}\right)$ (up to $\left.k\right)$ iff:

(i) for $i \leq k, a_{i}^{p}=a_{i}^{p^{\prime}}$; also $l_{i}^{p}=l_{i}^{p^{\prime}}$ when $i \in I_{\mathrm{afn}}$, and $T_{i}^{p}=T_{i}^{p^{\prime}}$ if $i \in I_{\text {fin }}$.

(ii) For each $i$ such that $\alpha(i) \leq k, T_{i}^{p} \subseteq T_{i}^{p^{\prime}}$.

(iii) Let $H=L\left(a_{0}\right) \cup L\left(a_{1}\right) \cup \cdots \cup L\left(a_{k}\right)$. Then $\operatorname{tp}(\bar{c} / H)=\operatorname{tp}\left(\bar{c}^{\prime} / H\right)$.

Claim. For each $i,\left(*_{i}\right)$ holds:

$\left(*_{i}\right)$ Given $p^{1}, p^{2}, \ldots \in \Pi$ and tuples $\bar{c}^{1}, \bar{c}^{2}, \ldots$ from $M$ of equal length, there exists an infinite $X \subset \omega$ and isomorphisms $h^{j}$ of $M$ for $j \in X$, such that if $j<j^{\prime} \in X$, then $h^{j}\left(p^{j}, \bar{c}^{j}\right) \leq{ }^{w} h^{j^{\prime}}\left(p^{j^{\prime}}, \bar{c}^{j^{\prime}}\right)$ (up to $i$ ), and $h^{j}\left(\bar{c}^{j}\right)=h^{j^{\prime}}\left(\bar{c}^{j^{\prime}}\right)$.

Proof. Assume inductively that $\left(*_{i-1}\right)$ holds. Let $\bar{d}^{j}$ be the concatenation of $\bar{c}^{j}, a_{i}^{j}, l_{i}^{j}$ if $i \in I_{\mathrm{afn}}$, and an enumeration of $T_{i}^{j}$ if $i \in I_{\mathrm{fin}}$. Applying $\left(*_{i-1}\right)$ to $p^{1}, p^{2}, \ldots$ and $\bar{d}^{1}, \bar{d}^{2}, \ldots$ we may assume that $p^{1} \leq^{w} p^{2} \leq^{w} p^{3} \leq^{w} \ldots$ (up to $i-1$ ), that all the $\bar{c}_{i}$ 's are equal to some $\bar{c}$, that $a_{i}^{j}=a_{i}, l_{i}^{j}=l_{i}$ (if $i \in I_{\text {afn }}$ ), and $T_{i}^{j}=T_{i}$ (if $i \in I_{\text {fin }}$ ). If $i \in I_{\text {fin }} \cup I_{\text {old }} \cup I_{\text {afn }}$ then it follows directly from the definition that $p_{j} \leq{ }^{w} p^{j^{\prime}}$ (up to $i$ ) whenever $j \leq j^{\prime}$. So the only case of interest is $i \in I_{\text {new }}$.

By Ramsey's theorem, it suffices to show that (in any infinite subsequence of the $p^{j}$ 's) one may find $j<j^{\prime}$ and an isomorphism $h$ of $M$ such that $h(\bar{c})=\bar{c}$ and $p^{j} \leq^{w} h\left(p^{j^{\prime}}\right)$ (up to $i$ ).

Let $H=L\left(a_{0}\right) \cup L\left(a_{1}\right) \cup \cdots \cup L\left(a_{i-1}\right)$. By definition of $I_{\text {new }}, P=L\left(a_{i-1}\right)$ is orthogonal to $H$. Moreover, we have assumed that each element of the underlying field of $P$ is definable over $a_{0}, \ldots, a_{i-1}$. It follows that every automorphism of $P$ over $a_{0}, \ldots, a_{i-1}$ is an automorphism over $H$. (One needs to know that every type of a tuple from $P$ is stationary over $a_{0}, \ldots, a_{i-1}$. See the proof of 3.11(d). Since the orderings $<_{i}^{j}$ of $P$ are of the same isomorphism type, we can find isomorphisms of $M$ fixing $H$ pointwise, and carrying one to the other. We may thus assume that $<_{i}^{j}$ is always the same standard ordering $<$ of $P$. In doing this we lose the assumption that $\bar{c}^{j}$ and $a_{i}^{j}$ do not depend on $j$, but we still have $\left(p^{j}, \bar{c}^{j}, a_{i}^{j}\right) \leq{ }^{w}\left(p^{j^{\prime}}, \bar{c}^{j^{\prime}}, a_{i}^{j^{\prime}}\right.$ ) (up to $i-1$ ) for $j<j^{\prime}$, since the automorphisms fix $H$. 
Let $\bar{e}^{1}$ be a tuple from $P$ and $\bar{e}$ a tuple from $H$ such that $\operatorname{tp}\left(\bar{c}^{1} / \bar{e}^{1} \bar{e}\right) \vdash$ $\operatorname{tp}\left(\bar{c}^{1} / H \cup P\right)$. They exist by $\omega$-stability, since $H \cup P$ is definable. Since each $\bar{c}^{j}$ is conjugate over $H$ to $\bar{c}^{1}$, we can find $\bar{e}^{j}$ for each $j$ so that $\operatorname{tp}\left(\bar{c}^{j} / \bar{e}^{j}\right) \vdash$ $\operatorname{tp}\left(\bar{c}^{j} / H \cup P\right) .\left(\bar{e}\right.$ and the length of $\bar{e}^{j}$ are constant.) Let $J=\left\{i^{\prime}: \alpha\left(i^{\prime}\right)=i\right\}$. For $i^{\prime} \in J, T_{i^{\prime}}^{j}$ is an initial section of $(P,<)$; say $T_{i^{\prime}}^{j}=\left\{x \in P: x<b_{i^{\prime}}^{j}\right\}$. Let $\bar{b}^{j}=\left(b_{i^{\prime}}^{j}: i^{\prime} \in J\right)$.

By Lemma 2.5, there exists $j<j^{\prime}$ and an order-preserving elementary map $h_{0}: P \rightarrow P$ with $h_{0}\left(\bar{e}^{j}\right)=\bar{e}^{j^{\prime}}$ and $h_{0}\left(\bar{b}^{j}\right)=\bar{b}^{j^{\prime}}$. So $h_{0}\left(T_{i^{\prime}}^{j}\right) \subset T_{i^{\prime}}^{j^{\prime}}$ for each $i^{\prime} \in J$. As the $T_{i^{\prime}}^{j}$ 's are finite, one can find an isomorphism $h$ of $P$ over $\left(a_{0}, \ldots, a_{i-1}\right)$ (not order-preserving) such that $h$ and $h_{0}$ agree on the $T_{i^{\prime}}^{j}$ 's $\left(i^{\prime} \in J\right), a_{i}^{j}$ and $\bar{e}^{j}$. By a remark made earlier, $h$ may be extended to an isomorphism of $M$ fixing $H$ pointwise. So

$$
\left(h\left(p^{j}\right), h\left(\bar{c}^{j}\right), h\left(a_{i}^{j}\right)\right) \leq^{w}\left(p^{j^{\prime}}, \bar{c}^{j^{\prime}}, a_{i}^{j^{\prime}}\right) \quad(\text { up to } i-1) .
$$

It follows that

$$
\left(h\left(p^{j}\right), h\left(\bar{c}^{j}\right), h\left(a_{i}^{j}\right)\right) \leq^{w}\left(p^{j^{\prime}}, \bar{c}^{j^{\prime}}, a_{i}^{j^{\prime}}\right) \quad(\text { up to } i) .
$$

Conditions (i) and (ii) are immediate from what has just been done. Condition (iii) reads $\operatorname{tp}\left(h\left(\bar{c}^{j}\right) / H \cup P\right)=\operatorname{tp}\left(\bar{c}^{j^{\prime}} / H \cup P\right)$. This follows from the fact that $h(\bar{e})=\bar{e}$ and $h\left(\bar{e}^{j}\right)=h_{0}\left(\bar{e}^{j}\right)=\bar{e}^{j^{\prime}}$, together with the choice of $\bar{e}^{j}, \bar{e}$. This proves the claim except for $h\left(\bar{c}^{j}\right)=\bar{c}^{j^{\prime}}$. By condition (iii) there exists an automorphism fixing $H \cup P$ and carrying $h\left(\bar{c}^{j}\right)$ to $\bar{c}^{j^{\prime}}$. Composing with it finishes the proof of the claim. The lemma follows by applying the claim with $i=n$ and all the $\bar{c}^{j}$ equal to an enumeration $\bar{c}$ of $F$.

We proceed to find a set of axioms for the theory of $M$. We first define an appropriate language $L$, using a slight refinement of (C3).

Fact 2.11. (a) There exists an integer $\lambda \geq n+1$ such that for any $U \in \mathscr{W}$, if $A$ is the base of $U$ then $\operatorname{tp}_{\lambda}(A) \vdash \operatorname{tp}(A) .\left(\operatorname{tp}_{\lambda}(Y)=\bigcup\left\{\operatorname{tp}\left(Y_{0}\right): Y_{0} \subset Y, \operatorname{card}\left(Y_{0}\right) \leq\right.\right.$ $\lambda+1\}$.)

(b) There exists an integer $\mu=\mu(2 \lambda)$ such that for any $2 \lambda$ elements of any $U \in \mathscr{W}$ lie in some $U^{\prime} \in \mathscr{W}$ with $U^{\prime} \subset U$, and such that the base of $U^{\prime}$ has at most $\mu$ elements. The base of $U^{\prime}$ can be chosen to have the same bounded part as the base of $U$, and hence base $\left(U^{\prime}\right) \subset$ base $(U)$.

Proof. We need the following variation of Lemma 2.4. (a) follows from it in much the same way as 2.4 gave (C3) (with $\lambda=\lambda(1)$ ), but a little more care is needed at limits. Let a section of a base $\left\{a_{0}\right\} \cup S_{1} \cup\left\{a_{1}\right\} \cup \cdots \cup S_{k}$ be a subset of the form $\left\{a_{0}\right\} \cup S_{1} \cup \cdots \cup S_{i}$ or $\left\{a_{0}\right\} \cup S_{1} \cup \cdots \cup S_{i} \cup\left\{a_{i}\right\}$. Then the correct inductive hypothesis is this: for each $p \in \Pi$, each section $X$ of the base of $U(p)$ satisfies: $\operatorname{tp}_{\lambda}(X) \vdash \operatorname{tp}(X)$. This goes through at limit steps because if $p_{\alpha x} \rightarrow p$ in $\Pi$, then for all sufficiently large $\alpha$, the base of $U(p)$ is a section of the base of $U\left(p_{\alpha}\right)$. 
Lemma 2.4'. For each integer $k$ there exists an integer $\lambda(k)$ such that for all $U \in \mathscr{W}$ and all k-element subsets $F$ of $M$, there exists a subset $H^{*}$ of $U$ of cardinality at most $\lambda(k)$ such that with $H=H^{*} \cap$ base $(U)$,

(i) $F \cap U \subset H^{*}, F \perp U \mid H^{*}$ and $\operatorname{tp}(F / S)$ is the unique nonforking extension to $S$ of $\operatorname{tp}\left(F / H^{*}\right)$.

(ii) $\operatorname{tp}(F / \operatorname{base}(U))$ is the unique nonforking extension of $\operatorname{tp}(F / H)$.

Proof. Fix $F, i$, and an $i$-tuple $\bar{a}$. Let $\mathscr{W}(\bar{a})=\{U \in \mathscr{W}:$ the bounded part of base $(U)$ is $\bar{a}\}$. For $U \in \mathscr{W}(\bar{a})$ let $\lambda(\bar{a}, F, U)$ be the least integer $\lambda$ such that there exists an $H^{*}$ of cardinality $\lambda$ satisfying (i) and (ii). One proves that $\lambda$ is bounded as in Lemma 2.4, but taking the embeddings over $\bar{a} \cup F$. (The point is that if $U^{1} \subset U^{2} \subset \cdots$ and each $U^{i}$ is in $\mathscr{W}(\bar{a})$, then by $\left(C 4^{\#}\right)$ base $\left(U^{1}\right) \subseteq$ base $\left(U^{2}\right) \subseteq \cdots$, so by $\omega$-stability, there exist $j<j^{\prime}<\omega$ such that $\operatorname{tp}\left(F / \operatorname{base}\left(U^{j^{\prime}}\right)\right)$ is the unique nonforking extension of $\operatorname{tp}\left(F / \operatorname{base}\left(U^{j}\right)\right)$ and $\operatorname{tp}\left(F / U^{j}\right)$ is the unique nonforking extension of $\operatorname{tp}\left(F / U^{j^{\prime}}\right)$.) $\lambda$ apparently depends on $\bar{a}$ and $F$, but as $\bar{a}$ is at most an $n$-tuple and there are finitely many $n+k$-types, it depends on $k=\operatorname{card}(F)$ alone.

(b) is proved in exactly the same way, letting $\mu(F, \bar{a}, U)$ be the least integer $\mu$ such that for some $U^{\prime} \in \mathscr{W}(\bar{a}), U^{\prime} \subseteq U, F \subseteq U^{\prime}$, and the base of $U^{\prime}$ has at most $\mu$ elements. Then $\mu(F, \bar{a}, U)$ is bounded for fixed $F$ and $\bar{a}$, and one lets $\mu(2 \lambda)=\sup \{\mu(F, \bar{a}, U): \operatorname{card}(F) \leq 2 \lambda\}$.

Let $\lambda=\lambda(1)$ and $\mu=\mu(2 \lambda)$. Choose a finite language $L$ for $M$ such that every atomic relation of $L$ has arity $\lambda+1$, and every definable relation of arity $\lambda+1$ is equivalent to an atomic relation of $L$. The only function symbols of $L$ are to be the restriction maps $r_{i+1} \rightarrow r_{j}$. This automatically gives a tree structure on any model $\widetilde{M}$ of $L$. (The loci of the $r_{i}$ are the only sorts, so they give a partition of $\widetilde{M}$, and one can define a partial ordering by: $x \leq y$ iff $x \in r_{i}, y \in r_{j}, j \geq i$, and $x$ is the image of $y$ under the restriction map from $r_{j}$ to $r_{i}$.) So we can define $\mathscr{W}_{0}(\widetilde{M})$, and the base of an element of $\mathscr{W}_{0}(M)$, as in Definition 2.7. Note that $\left(\mathrm{C}^{\#}\right)$ holds for $\mathscr{W}_{0}(\widetilde{M})$.

Axiom Group I. State the cardinality of finite sets definable with $\leq \lambda$ elements. For every pair of atomic formulas $\varphi(x, \bar{y})$ and $\psi(\bar{y})$ (so length $(\bar{y}) \leq \lambda)$, if $M \Vdash(\forall \bar{y})\left(\psi(\bar{y}) \rightarrow\left(\exists^{! m} x\right) \varphi(x, \bar{y})\right)$ for some $m$, then this sentence is an axiom. Axiom Group II. $L\left(a_{1}, \ldots, a_{i-1}\right)$ is infinite $\left(i \notin I_{\text {fin }}\right)$.

Axiom Group III. Suppose $U \in \mathscr{W}$ has a base $A$ of cardinality at most $\mu$, and $\bar{b}$ is a $\lambda$-tuple from $U$. Let $q, r$ be partial $\bar{b}$-definable atomic types over $U$. This means that there exists a function $\varphi(x, \bar{y}) \mapsto\left(d_{q} x\right) \varphi(x, \bar{y}, \bar{z}, A)$ from atomic formulas to formulas, such that $q$ has the form

$$
q=\left\{\varphi(x, \bar{c}): \bar{c} \text { from } U, \text { and } \vDash\left(d_{q} x\right) \varphi(x, \bar{c}, \bar{b}, A)\right\}
$$

(and similarly for $r$ ). Assume $q \vdash r$. We will add an axiom to describe this situation. 
$U$ is a definable set in $M$, and hence can be considered as an $\aleph_{0}$-categorical structure in its own right, with the full structure induced on it by $M$. Define a $U$-restricted formula to be a formula obtained from the atomic relations using Boolean operations and the quantifiers: $(\exists x \in U),(\forall x \in U)$. By (C3), every relation definable on $U$ as a subset of $M$ is in fact definable from $U$ with the $L$-structure alone; i.e. it is equivalent to a $U$-restricted formula. In particular, for each atomic $\varphi(x, \bar{y})$, there exists a $U$-restricted formula $\varphi_{q}^{*}(\bar{y}, \bar{z}, A)$ equivalent to $\left(\bar{y}, \bar{z} \in U\right.$ and $\left.\left(d_{q} x\right) \varphi(x, \bar{y}, \bar{z}, A)\right)$ (similarly for $r$ ). In addition, there exists a $U$-restricted formula $\rho(\bar{z}, A)$ that isolates $\operatorname{tp}(\bar{b} / A)$.

By Fact 2.11, the atomic type of $A$ implies the full type of $A$. It is therefore the case that for every $U^{\prime} \in \mathscr{W}_{0}(M)$, if the base $A^{\prime}$ of $U^{\prime}$ realizes the atomic type of $A$, then for all $\bar{b}^{\prime} \in U^{\prime}$, if $\models \rho\left(\bar{b}^{\prime}, A\right)$ then for all $x \in M$, if for every atomic $\varphi, \varphi(x, \bar{c})$ holds for every $\bar{c}$ such that $\vDash \varphi_{q}^{*}\left(\bar{c}, \bar{b}^{\prime}, A^{\prime}\right)$, then for every atomic $\varphi, \varphi(x, \bar{c})$ holds for every $\bar{c}$ such that $\models \varphi_{q}^{*}\left(\bar{c}, \bar{b}^{\prime}, A^{\prime}\right)$. This is a single sentence which we call an axiom. Note that altogether we have finitely many such.

We need to prove that this axiomatizes $M$ in the language $L$. Let $\widetilde{M}$ be another model of the axioms. We will build an isomorphism from $M$ to $\widetilde{M}$. This will be done by induction. We will find a continuous, increasing sequence $p(\alpha)$ of elements of $\Pi$, and a continuous chain of maps $h_{\alpha}: U_{p(\alpha)} \rightarrow$ $\widetilde{M}$, satisfying:

(i) The $h_{\alpha}$ preserve the atomic relations of $L$.

(ii) The image under $h_{\alpha}$ of $U_{p(\alpha)}$ is in $\mathscr{W}_{0}(\widetilde{M})$, and the base of the image of $U_{p(\alpha)}$ is the image of the base of $U_{p(\alpha)}$.

In particular, it follows from (ii) that if $U_{p(\alpha)}=M$, then the image of $M$ under $h_{\alpha}$ is the unique member of $\mathscr{W}_{0}(\widetilde{M})$ whose base is the singleton set consisting of the root of the tree, i.e. $\widetilde{M}$ itself. Thus $h_{p(\alpha)}$ will be an isomorphism. So we only need to show that the chain can be continued until the domain becomes $M$.

Note that (i) is trivial at limit stages, and (ii) at successor stages. We first deal with the successor case. The image under $h$ of an object $X$ associated with $M$ will be denoted $\widetilde{X}$.

Claim 2.12. Let $h: U \rightarrow \widetilde{M}$ satisfy (i) and (ii), where $U=U(p), p \in \Pi$, $\operatorname{base}(U)=\left\{a_{0}\right\} \cup S_{1} \cup \cdots \cup\left\{a_{k-1}\right\} \cup S_{k}$. Suppose $p^{+} \in \Pi, p^{+} \geq p, U^{+}=U\left(p^{+}\right)$, $\operatorname{base}\left(U^{+}\right)=\left\{a_{0}\right\} \cup S_{1} \cup \cdots \cup\left\{a_{k-1}\right\} \cup S_{k} \cup\left\{a_{k}\right\} \cup \varnothing$ or $k=n$, $\operatorname{base}\left(U^{+}\right)=$ $\left\{a_{0}\right\} \cup S_{1} \cup \cdots \cup\left\{a_{k-1}\right\} \cup S_{k}^{+}, S_{k}^{+}=S_{k} \cup\left\{a_{k}\right\}$. Then $h$ extends to a map on $U^{+}$satisfying (i) and (ii).

Proof. Consider $\operatorname{tp}\left(a_{k} / U\right)$. By stability it is a definable type. Let $r$ be the set of atomic formulas in this type. By Lemma 2.4, there exists a $\lambda$-tuple $\bar{b}$ 
from $U$ such that $\operatorname{tp}\left(a_{k} / U\right)$ is the unique nonforking extension of $\operatorname{tp}\left(a_{k} / \bar{b}\right)$. It follows directly that $r$ is $\bar{b}$-definable

Let $\varphi(x, \bar{b})$ be an atomic formula isolating $\operatorname{tp}\left(a_{k} / \bar{b}\right)$. Let $q(x)=\{\varphi(x, \bar{b})\}$ if $\operatorname{tp}(x / \bar{b})$ is algebraic. If $\operatorname{tp}(x / \bar{b})$ is strongly minimal, let $q(x)=\{\varphi(x, \bar{b})\} \cup$ $\{\sim \theta(x, \bar{c}): \bar{c}$ a $\lambda$-tuple from base $(U)$, and $\theta(x, \bar{c})$ is an atomic formula with only finitely many solutions $\}$. In this case we can simply choose $\bar{b}=a_{k-1}$, $\varphi(x, \bar{b})=\left(x \in L\left(a_{1}, \ldots, a_{k-1}\right)\right) . q$ is a $\bar{b}$-definable atomic type in either case. We will show that $q \vdash r$ and $\tilde{q}$ is realized in $\widetilde{M}$. An axiom in group III implies that any realization of $\tilde{q}$ in $\widetilde{M}$ realizes $\tilde{r}$. This proves that $h$ can be extended.

(The statement before the last may require clarification. Consider sets $U^{\prime} \subseteq$ $U, U^{\prime} \in \mathscr{W}$ whose base has cardinality $\leq \mu$ and the same bounded part as base $(U)$, and with $\bar{b} \subseteq U^{\prime}$. The proof that $q \vdash r$ will of course show that $q\left|U^{\prime} \vdash r\right| U^{\prime}$ for any such $U^{\prime}$. So some axiom from group III will state that the type over $\widetilde{U}^{\prime}$ defined by the image of the definition of $q$ under $h$ implies the type over $\widetilde{U}^{\prime}$ defined by the image of the definition of $r$. Since the definitions are in terms of restricted formulas, and $h$ is at any rate an isomorphism of $\widetilde{U}$ with $\widetilde{U}^{\prime}$, the types in question are $\tilde{q} \mid \widetilde{U}^{\prime}$ and $\tilde{r} \mid \widetilde{U}^{\prime}$, respectively. So $q\left|\widetilde{U}^{\prime} \vdash \tilde{r}\right| \widetilde{U}^{\prime}$. By $2.11(\mathrm{~b}), r=\bigcup\left\{r \mid U^{\prime}: U^{\prime}\right\}$, so $\tilde{r}=\bigcup\left\{\tilde{r} \mid \widetilde{U}^{\prime}: U^{\prime}\right\}$. Letting $U^{\prime}$ vary we get $\left.\tilde{q} \vdash \tilde{r}_{\text {. }}\right)$

In the algebraic case both claims are clear. So assume we are in the strongly minimal case. Let $X=L\left(a_{1}, \ldots, a_{k-1}\right) \cap \operatorname{acl}(U) . X$ is a base $(U)$-definable subset of $L\left(a_{1}, \ldots, a_{k-1}\right)$, so it is either finite or cofinite. In the second case, the finite subset of $L\left(a_{1}, \ldots, a_{k-1}\right)$ missed by $X$ is $\subset \operatorname{acl}(\operatorname{base}(U))$; since $X \supset \operatorname{acl}(\operatorname{base}(U))$, we must have $X=L\left(a_{1}, \ldots, a_{k-1}\right)$. But this contradicts the fact that $\operatorname{tp}\left(a_{k} / U\right)$ is not algebraic. Thus $X$ is finite; being base $(U)$-definable it is contained in $\operatorname{acl}(\operatorname{base}(U))$; so $X=\operatorname{acl}(\operatorname{base}(U))$. Now if $a \models q$ then by Lemma $2.4^{\prime} a$ is not algebraic over base $(U)$. It follows that $a \notin \operatorname{acl}(U)$, so $a \models r$. This shows $q \vdash r$. The fact that $\tilde{q}$ is realized in $\widetilde{M}$ is obvious from Axiom group II.

It remains to deal with the limit case. It is clear that this has no chance of working if the choices in the successor case are carried out randomly. So fix in advance a well-ordering of $\widetilde{M}$ of order type $\omega$. Each time one has a choice about extending $h_{\alpha}$, make the least possible one.

Fact 2.13. Let $p_{\alpha}$, and $h_{\alpha}$ be defined for $\alpha<\alpha^{*}$, satisfying (i) and (ii). Let $p$ be the least upper bound of the $p_{\alpha}$ 's, and let $h$ be the union of the $h_{\alpha}$ 's. Assume the $h_{\alpha}$ 's have been defined as stated in the previous paragraphs for successor $\alpha<\alpha^{*}$. Then $p$ and $h$ satisfy (i) and (ii).

Proof. Recall the proof of $(\mathrm{C} 1)$. Say $p_{\alpha}=\left(a_{0}^{\alpha}, S_{1}^{\alpha}, \ldots, a_{k(\alpha)}^{\alpha}, S_{k(\alpha)}^{\alpha} ; \cdots\right)$. Let $i$ be the largest integer such that $a_{0}^{\alpha}, S_{1}^{\alpha}, \ldots, S_{i-1}^{\alpha}, a_{i-1}^{\alpha}$ eventually settle down (say to $\left.a_{0}, S_{0}, \ldots, S_{i-1}, a_{i-1}\right)$. Then $L\left(a_{1}, \ldots, a_{i-1}\right) \subset U(p)$, and the only problem is to show that $L\left(\tilde{a}_{1}, \ldots, \tilde{a}_{i-1}\right) \subset \widetilde{U}(p)$. 
Note first that $L\left(\tilde{a}_{1}, \ldots, \tilde{a}_{i-1}\right) \cap \operatorname{acl}_{L}(\tilde{U}(p))=L\left(\tilde{a}_{1}, \ldots, \tilde{a}_{i-1}\right) \cap \tilde{U}(p)$. For if $\tilde{x} \in L\left(\tilde{a}_{1}, \ldots \tilde{a}_{i-1}\right)$ and $\widetilde{M} \models \varphi(\tilde{x}, \tilde{c})$, where $\varphi$ is atomic and algebraic and $\tilde{c}$ is a $\lambda$-tuple from $\tilde{U}(p)$, then we have $\tilde{c}=h(\bar{c})$ for some $\lambda$-tuple $\bar{c}$ from $U(p) . \varphi(x, \widetilde{c})$ has exactly $m$ solutions for some $m$. All of these solutions lie in $U\left(p_{\alpha}\right)$ for some $\alpha<\alpha^{*}$. By an axiom in group I, $\varphi(x, \tilde{c})$ has precisely $m$ solutions in $\widetilde{M}$. It follows that all $m$ lie in $\widetilde{U}\left(p_{\alpha}\right)$, hence in $\widetilde{U}(p)$.

Now distinguish two cases. It may be that for some $\alpha<\alpha^{*}, L\left(a_{1}, \ldots, a_{i-1}\right)$ $\subset \operatorname{acl}\left(U\left(p_{\alpha}\right)\right)$. In this case we are immediately finished. So assume otherwise. Let $\tilde{a}$ be the least element of $L\left(\tilde{a}_{1}, \ldots, \tilde{a}_{i-1}\right)-\widetilde{U}(p)$. So all the preceding elements are in $\tilde{U}\left(p_{\alpha_{0}}\right)$ for some $\alpha_{0}<\alpha^{*}$. Choose $\alpha_{0}$ large enough so that for $\alpha \geq \alpha_{0}, p_{\alpha}=\left(a_{0}, S_{0}, \ldots, S_{i-1}, a_{i-1}, S_{i}^{\alpha}\right.$, etc. $)$ for some $S_{i}^{\alpha}$. Let $X_{0}=\operatorname{acl}\left(S_{0} \cup\right.$ $\left.\cdots \cup S_{i-1}\right)$, and let $T^{\alpha}$ be the tree-upward-closure of $S_{i}^{\alpha}$. Then base $\left(U\left(p_{\alpha}\right)\right) \subseteq$ $X_{0} \cup T^{\alpha} \subseteq\left(p_{\alpha}\right)$. Since $L\left(a_{1}, \ldots, a_{i-1}\right) \nsubseteq \operatorname{acl}\left(U\left(p_{\alpha}\right)\right), T^{\alpha}$ must be orthogonal to $L\left(a_{1}, \ldots, a_{i-1}\right)$. So

$$
L\left(a_{1}, \ldots, a_{i-1}\right) \cap \operatorname{acl}\left(X_{0} \cup T^{\alpha}\right) \subseteq \operatorname{acl}\left(X_{0} \cup S_{i}^{\alpha}\right) .
$$

It follows that if $\alpha$ is the least ordinal $>\alpha_{0}$ such that $U\left(p_{\alpha+1}\right)=U\left(p_{\alpha}\right) \cup\{w\}$ with $w \in L\left(a_{1}, \ldots, a_{i-1}\right)$ and $w \notin \operatorname{acl}\left(X_{0} \cup S_{i}^{\alpha_{0}}\right)$, then $w \notin \operatorname{acl}\left(X_{0} \cup S_{i}^{\alpha}\right)$, so $w \notin \operatorname{acl}\left(\operatorname{base}\left(U\left(p_{\alpha}\right)\right)\right.$. In this case the proof of the successor case called for extending $h_{\alpha}$ to $h_{\alpha+1}$ by sending $w$ to the least element of $L\left(\tilde{a}_{1}, \ldots, \tilde{a}_{i-1}\right)$ $\operatorname{acl}_{L}\left(\operatorname{base}\left(\tilde{U}\left(p_{\alpha}\right)\right)\right)$. This least element must clearly be $\tilde{a}$, so $\tilde{a}$ is in the range after all.

This finishes the proof. We conclude with a curious characterization of $\omega$ stability, leaving the proof to the reader.

Fact 2.14. Let $T$ be a countable theory, $\alpha$ a countable ordinal. Then TFAE:

(i) $T$ has Morley rank $<\alpha$.

(ii) For every system $(\Pi, U)$ satisfying $(C 1)$ and $(C 4),\left(\omega^{\alpha},<\right)$ does not embed into $(\Pi,<)$.

Proof of Lemma 2.5. It is clearly stronger to prove the lemma for a vector space than for projective space. Let $V$ be a vector space over a finite field $F$ with basis $v_{0}, v_{1}, \ldots$. Fix a linear ordering of $F$ with 0,1 as the first two elements. Order $V$ lexicographically: $\sum \alpha_{i} v_{i}<\sum \beta_{i} v_{i}$ iff for some $j, \alpha_{i}=\beta_{i}$ for $i>j$ and $\alpha_{i}<\beta_{i}$.

Lemma. Let $\bar{x}_{p}=\left(x_{p}^{1}, \ldots, x_{p}^{n}\right)$ be an n-tuple of elements of $V$ for $p=$ $1,2, \ldots$ Then for some $p_{0}<p_{1}$ there exists an order preserving linear embedding $h: V \rightarrow V$ with $h\left(\bar{x}_{p_{0}}\right)=\left(\bar{x}_{p_{1}}\right)$.

Proof. For any $u=\sum \alpha_{i} v_{i}$ in $V$, let $\operatorname{supp}(u)=\left\{i: \alpha_{i} \neq 0\right\}$, and let lead $(u)=$ $\sup (\operatorname{supp}(u))$. Let $U_{p}$ be the subspace of $V$ generated by $\left\{x_{p}^{1}, \ldots, x_{p}^{n}\right\}$. Choose a basis $u_{p}^{1}, \ldots, u_{p}^{n^{\prime}}$ for $U_{p}$, with $\operatorname{lead}\left(u_{p}^{1}\right)<\cdots<\operatorname{lead}\left(u_{p}^{n^{\prime}}\right)$. We 
may write $\bar{x}_{p}=M_{p} \bar{u}_{p}$, where $M_{p}$ is an $n \times n^{\prime}$-matrix over $F$. For infnitely many $p, M_{p}$ equals a fixed matrix $M$; throw out the others.

We will use the following result from [Hi]: let $w_{p}$ be a word in a finite alphabet for $p<\omega$. Then there exist $p_{1}<p_{2}$ such that $w_{p_{1}}$ is a subword of $w_{p_{2}}$, i.e. $w_{p_{1}}$ can be obtained by deleting some letters from $w_{p_{2}}$. Associate a word $w_{p}$ to each $u_{p}$; the $j$ th letter in this word will give the $v_{j}$-coefficient of $u_{p}^{k}$ for $k \leq n^{\prime}$, as well as the information of whether or not lead $\left(u_{p}^{k}\right)=j$. (To prevent the word from being infinite, cut it off after the largest lead $\left(u_{p}^{k}\right)$.) By Higman's lemma, there exist $p_{0}<p_{1}$ and a nondecreasing map $\varepsilon: \omega \rightarrow \omega$ with the following property: if $l_{i}=\operatorname{lead}\left(u_{p_{0}}^{i}\right), m_{i}=\operatorname{lead}\left(u_{p_{1}}^{i}\right), u_{p_{0}}^{i}=\sum_{j \leq l_{i}} \alpha_{j}^{i} v_{j}$ and $u_{p_{1}}^{i}=\sum_{j \leq l_{i}^{\prime}} \beta_{j}^{i} v_{j}$, then $\varepsilon\left(l_{i}\right)=m_{i}$ and $\alpha_{j}^{i}=\beta_{\varepsilon(j)}^{i}$ for each $i, j$.

Now define a linear map $h: V \rightarrow V$ by specifying $h\left(v_{p}\right)$ for each $p . h$ will be order-preserving because it will satisfy:

$(*) \quad h\left(v_{p}\right)=v_{\varepsilon(p)}+r_{p}, \quad$ where lead $\left(r_{p}\right)<\varepsilon(p)$ and $\operatorname{supp}\left(r_{p}\right) \cap \operatorname{range}(\varepsilon)=\varnothing$.

For any such $h$,

$$
\begin{aligned}
h\left(u_{p_{0}}^{i}\right) & =h\left(\sum_{j \leq l_{i}} \alpha_{j}^{i} v_{j}\right)=\sum_{j \leq l_{i}} \alpha_{j}^{i}\left(v_{\varepsilon(i)}+r_{j}\right) \\
& =\sum_{j \leq l_{i}} \alpha_{j}^{i} v_{\varepsilon(i)}+\sum_{j \leq l_{i}} \alpha_{j}^{i} r_{j}=\sum_{j \in J} \beta_{j}^{i} v_{j}+\sum_{j \leq l_{i}} \alpha_{j}^{i} r_{j},
\end{aligned}
$$

where $J=\left\{j \leq m_{i}: j \in \operatorname{range}(\varepsilon)\right\}$. So we will have $H\left(\bar{u}_{p_{0}}\right)=\bar{u}_{p_{1}}$ provided that for each $i, \sum_{j \leq l_{i}} \alpha_{j}^{i} r_{j}=\sum_{j \notin J} \beta_{j}^{i} v_{j}$. We will choose $r_{j}=0$ unless $j=l_{i}$ for some $i \leq n^{\prime}$; letting $w_{i}=\sum_{j \notin J} \beta_{j}^{i} v_{j}$, the requirement then becomes: $\sum_{i \leq k} \alpha_{l_{i}}^{k} r_{l_{i}}=w_{k}$ for each $k \leq n^{\prime}$. The matrix $\left(\alpha_{l_{i}}^{k}\right)$ is triangular with no zeros on the diagonal $\left(\alpha_{l_{i}}^{i} \neq 0\right.$ by definition of $\left.l_{i}=\operatorname{supp}\left(u_{p}^{i}\right)\right)$, so one can solve for the $r_{l_{i}}$. Moreover, $r_{l_{i}}$ will be in the span of $w_{1}, \ldots, w_{i}$, so we will have $\operatorname{supp}\left(r_{l_{i}}\right) \cap J=\varnothing$ and $\operatorname{lead}\left(r_{l_{i}}\right) \leq \max _{k \leq i} \operatorname{lead}\left(w_{k}\right)<m_{i}$, so the requirements in $(*)$ are satisfied. Thus there exists an order-preserving linear map $h: V \rightarrow V$ satisfying $h\left(\bar{u}_{p_{0}}\right)=\bar{u}_{p_{1}}$. Multiplying by $M$, it follows that $h\left(\bar{x}_{p_{0}}\right)=\bar{x}_{p_{1}}$, ending the proof.

\section{EXPANDING TO A FINITE-DIMENSIONAL STRUCTURE}

Theorem 3.1. Let $M$ be $\aleph_{0}$-stable and $\aleph_{0}$-categorical. Then $M$ is a reduct of an $\aleph_{0}$-stable and $\aleph_{0}$-categorical, nonmultidimensional model $\widetilde{M}$. In fact, $\widetilde{M}$ can be chosen to have 0-definable strictiy minimal sets $D_{1}, \ldots, D_{n}$, with $D_{i}$ a projective geometry over a finite prime field $F_{i}$, and no other dimensions. The fields $F_{i}$ can be chosen to have distinct characteristics.

(It is easy to see that two projective spaces of distinct characteristics cannot live inside the same totally categorical theory, so the number of dimensions cannot be reduced any further.) 
Notation. $\operatorname{Aut}_{M}(D / F)$ is the group of all permutations of $D$ that extend to an automorphism of $M$ fixing $F$. Here $D$ is any set, not necessarily definable.

Definitions 3.2. (a) Let $D_{1}, D_{2}$ be definable sets in a structure $M . D_{1}$ is $D_{2}$ internal if there exists $n$ and a surjective definable function $f: D_{2}^{n} \rightarrow D_{1}$. The definition of $f$ may use parameters.

(b) $D_{1}$ is $D_{2}$-analyzable if in every elementary extension $\widehat{M}$ of $M$ there exists a sequence $E_{0}, \ldots, E_{m}$ of definable equivalence relations on $D_{1}$ such that $E_{0}=\left\{(d, d): d \in D_{1}\right\}, E_{m}=D_{1} \times D_{1}, E_{i}$ refines $E_{i+1}$ for each $i$, and for each $E_{i+1}$-class $C, C / E_{i}$ is $D_{2}$-internal.

(c) A partial type $\Phi$ is $D_{2}$-internal (analyzable) if the set defined by some formula in it has this property.

Lemma 3.3. If $M$ is sufficiently saturated, then:

(a) $D_{1}$ is $D_{2}$-internal iff for every elementary submodel $K \subset M$ over which $D_{1}, D_{2}$ are defined, $D_{1} \subseteq \operatorname{dcl}\left(K \cup D_{2}\right)$; iff there exists a finite $E$ such that $D_{1} \subseteq \operatorname{dcl}\left(E \cup D_{2}\right)$.

(b) $D_{1}$ is $D_{2}$-analyzable iff for each $a \in D_{1}$ there exist $a_{1}, \ldots, a_{n} \in \operatorname{dcl}(a)$ such that $a=a_{n}$, and $\operatorname{tp}\left(a_{i} /\left\{a_{1}, \ldots, a_{i-1}\right\}\right)$ is $D_{2}$-internal, for each $i$.

Proof. Easy, using compactness.

Claim 3.4. (a) If $D_{1}$ is $D_{2}$-analyzable and $D_{2}$ is $D_{3}$-analyzable then $D_{1}$ is $D_{3}$-analyzable; provided that $T$ is stable.

(b) If $D_{1}$ is finite and $D_{2}$ has cardinality at least 2, then $D_{1}$ is $D_{2}$-internal.

(c) If $D_{1}$ is strongly minimal and $D_{1} \not D_{2}$, then $D_{1}$ is $D_{2}$-analyzable.

(d) If $M$ is a reduct of $M^{\prime}, D_{1}, D_{2}$ are definable subsets of $M$, and $D_{1}$ is $D_{2}$-analyzable in $M$, then the same is true in $M^{\prime}$.

Proof. Only (a) is nontrivial, and it becomes so using the following characterization from $[\mathrm{H}]: \operatorname{tp}(a / B)$ is $D$-analyzable iff for every $X, \operatorname{if} \operatorname{tp}(a / X)$ is orthogonal to $D$ then $a \in \operatorname{acl}(X)$.

Definition 3.5. If $D$ is a definable set in a theory $T$, call $D \aleph_{0}$-stable $\left(\aleph_{0}\right.$ categorical) if for every uncountable cardinal $\kappa$ (for $\kappa=\aleph_{0}$ ), every elementary extension $\widehat{M}$ of $M$, and every $A \subset \widehat{M}$ with $\operatorname{card}(A)<\kappa$, there are fewer than $\kappa$ types in $D$ over $A$.

Warning. The definition of " $D$ is $\aleph_{0}$-categorical" may depend on the structure of the model outside $D$, even if $T$ is stable.

Lemma 3.6. Let $D_{1}, D_{2}$ be definable subsets of a structure $M . D_{1}$ is $\aleph_{0}$ stable $\left(\aleph_{0}\right.$-categorical $)$, and $D_{2}$ is $D_{1}$-analyzable. Then $D_{2}$ is $\aleph_{0}$-stable $\left(\aleph_{0}\right.$ categorical ).

Proof. We may assurne $M$ is quite saturated. The lemma then follows by induction from the following two claims. 
Claim 3.7. Suppose $M$ is $\aleph_{1}$-saturated.

(a) If $D_{1}$ is $D_{2}$-internal and $D_{2}$ is $\aleph_{0}$-stable ( $\aleph_{0}$-categorical), then so is $D_{1}$.

(b) If $E$ is a definable equivalence relation on $D, D / E$ is $\aleph_{0}$-stable ( $\aleph_{0}$ categorical) and each $E$-class is $\aleph_{0}$-stable ( $\aleph_{0}$-categorical), then so is D.

Proof. By counting types.

(a) Let $f: D_{2}^{n} \rightarrow \rightarrow D_{1}$ be a surjective definable function. Say $f$ is $C_{0^{-}}$ definable, $C_{0}$ finite. Then for every set $C \supseteq C_{0}$, there are at most as many 1-types over $C$ inside $D_{2}$ as there are $n$-types in $D_{2}^{n}$.

(b) $\operatorname{tp}(a / C)$ is determined by $\operatorname{tp}((a / E) / C)$ and $\operatorname{tp}(a / C \cup\{(a / E)\})$.

Note that if $M$ is $D$-analyzable, then $M$ is minimal over $D^{M}$.

The following is trivial but convenient:

Lemma 3.8. Let $T$ be $\aleph_{0}$-categorical. Then $T$ is bi-interpretable with a theory $T^{\prime}$ with a unique 1-type.

Proof. Let $p_{1}, \ldots, p_{n}$ be the 1-types of $T$. Let $a_{i} \models p_{i}$. Let $a=\left(a_{1}, \ldots, a_{n}\right)$, $p=\operatorname{tp}(a), M^{\prime}=$ the locus of $p$, and $T^{\prime}=\operatorname{Th}\left(M^{\prime}\right)$.

Proof of 3.1. We will prove the theorem by induction on the following index, measuring how far $M$ is from being totally categorical. Define the essential rank and multiplicity of $M,(e-\operatorname{rk}(M), e$-mult $(M))$ as the smallest pair of integers $k, m$ such that $x=x$ is analyzable over a definable set (in $M^{\mathrm{eq}}$ ) of Morley rank $k$, multiplicity $m$.

Assume that every theory of index less than $k, m$ satisfies the conclusion of 3.1. Let $D_{0}$ be a set of Morley rank $k$, multiplicity $m$ such that $x=$ $x$ is $D_{0}$-analyzable. Let $D$ be a definable subset of $D_{0}$ of Morely rank $k$, multiplicity 1 ; and let $D^{\prime}=D_{0}-D$. Without loss of generality $D, D_{0}$ are definable over $\varnothing$. Let $a \in D, \operatorname{rk}(a / \varnothing)=k$. Find $a_{1}, \ldots, a_{k} \in \operatorname{acl}(a)$ such that $\operatorname{tp}\left(a_{i} /\left\{a_{1}, \ldots, a_{i-1}\right\}\right)$ is strongly minimal. Let $D_{1}=\operatorname{stp}\left(a_{1} \cdots a_{k-1} / \varnothing\right)$. Note that $D_{2}=D^{\prime} \cup D_{1}$ has smaller rank or multiplicity than $D_{0}$.

Find a strictly minimal, modular set $P=P_{b_{0}}$ (definable over $b_{0}$ ), such that $\operatorname{stp}\left(a / a_{1}, \ldots, a_{k-1}\right) \not \perp P, b_{0} \in \operatorname{acl}\left(a_{1}, \ldots, a_{k-1}\right)$, and the Morley rank of $b_{0}$ over $\varnothing$ is least possible.

Claim 3.9. If $b$ is conjugate to $b_{0}$, then $\operatorname{stp}\left(b / b_{0}\right) \perp P_{b_{0}}$.

Proof. Since the theory is 1-based, $b \perp b_{0} \mid c$, where $c=\operatorname{acl}(b) \cap \operatorname{acl}\left(b_{0}\right) \quad(\operatorname{acl}$ evaluated in $\left.M^{\mathrm{eq}}\right)$. If $\operatorname{stp}\left(b / b_{0}\right) \not \perp P$ then $\operatorname{stp}(b / c) \not \perp P$, so by the minimality of $\operatorname{rk}\left(b_{0}\right)$ it follows that $\operatorname{rk}(c)=\operatorname{rk}\left(b_{0}\right)$. Hence

$$
\operatorname{rk}(b / c)=\operatorname{rk}(b)-\operatorname{rk}(c)+\operatorname{rk}(c / b)=\operatorname{rk}\left(b_{0}\right)-\operatorname{rk}(c)+0=0 .
$$

So $\operatorname{stp}\left(b / b_{0}\right)$ is algebraic, hence certainly orthogonal to $P$. 
Hence $P$ is still strictly minimal over $\left\{b, b_{0}\right\}$. So if $P_{b} \not \subset P_{b}^{\prime}$ (where $b, b^{\prime}$ are conjugate to $b_{0}$ over $\varnothing$ ), then there exists a unique 0 -definable bijection between $P_{b}$ and $P_{b^{\prime}}$. Let $D_{3}$ be the set of conjugates of $b_{0}$, and define an equivalence relation on the set of conjugates of $b$ by $b \sim b^{\prime}$ iff $P_{b} \not P_{b^{\prime}}$. Then by using the above remarks, we can define, uniformly in $b / \sim$, a set $P^{\prime}(b / \sim)$ uniformly in $b$, such that for any $b$ conjugate to $b_{0}$, there exists a (unique) $b$-definable bijection between $P_{b}$ and $P^{\prime}(b / \sim)$. Thus, replacing $P$ by $P^{\prime}$ and $b_{0}$ by $b_{0} / \sim$, we may assume that $\sim$ is the identity on the conjugates of $b_{0}$; i.e. $b \neq b^{\prime} \Rightarrow P_{b} \perp P_{b^{\prime}}$. Let $D_{3}$ be the set of conjugates of $b_{0}$, and $\hat{D}=D_{2} \cup D_{3}$. Since $\operatorname{rk}\left(b_{0}\right)=k-1$, we have $(\operatorname{rk}(\hat{D}), \operatorname{mult}(\hat{D}))<\left(\operatorname{rk}\left(D_{0}\right), \operatorname{mult}\left(D_{0}\right)\right)$.

Claim 3.10. $M$ is $\bigcup\left\{P_{b}: b \in D_{3}\right\} \cup \hat{D}$-analyzable. $P_{b_{0}} \perp \hat{D}$.

Proof. The first statement is clear, using the fact that $\operatorname{stp}\left(a / a_{1}, \ldots, a_{k-1}\right)$ is strongly minimal and nonorthogonal to $\bigcup_{b} P_{b}$, and Lemma $3.4(\mathrm{a})$ and (b). Suppose $P_{b_{0}} \not \hat{D}$. By 3.4(c), $P_{b_{0}}$ is $\hat{D}$-analyzable. Since $\hat{D}$ is 0 -definable, $P_{b}$ is $\hat{D}$-analyzable for each $b \in D_{3}$. By 3.9 , the $P_{b}$ 's are pairwise disjoint; so one has a definable equivalence relation on $\bigcup_{b} P_{b}$ whose classes are the $P_{b}$ 's. It follows by Definition $3.2(\mathrm{~b})$ that $\bigcup_{b} P_{b}$ is $\hat{D}$-analyzable. By $3.3(\mathrm{a}), M$ is $\hat{D}$-analyzable. But then $(e-\operatorname{rk}(M), e-\operatorname{mult}(M))<(k, m)$, a contradiction.

Let $P^{*}$ be a structure such that each $P_{b} \quad\left(b \in D_{3}\right)$ is abstractly isomorphic to $P^{*}$. $P^{*}$ is either a structureless countable set or else a projective geometry over a finite field. In the former case, let $Q^{*}=P^{*}$ and ignore the following lemma.

Fact 3.11. Suppose $P^{*}$ is a projective geometry over the finite field $F$, and $F_{0} \subset F$ is a prime subfield. Then there exists a subset $Q^{*}$ of $P^{*}$ with the following properties.

(a) Let $R=\left\{(a, b, c) \in P^{*}: a, b, c\right.$ are dependent but distinct $\}$. Then $R \cap Q^{* 3}$ gives $Q^{*}$ the structure of an infinite dimensional vector space over $F_{0}$.

(b) Every automorphism of $\left(Q^{*}, R \cap Q^{* 3}\right)$ extends to an automorphism of $P^{*}$.

(c) Let $k=\left[F: F_{0}\right]$. Then every $a \in P^{*}$ lies in the projective $F$-space generated by some $k$-elements of $Q^{*}$.

(d) If $\bar{a}, \bar{b} \in Q^{* n}$ realize the same type (as elements of $P$ ), then they realize the same strong type.

Proof. The relationship between $P^{*}$ and $Q^{*}$ is the projective version of the relationship between $V=U \otimes_{F_{0}} F$ and $U$, where is an infinite dimensional $F_{0}$-space. In more detail: Choose a vector space $U$ over $F_{0}$, with $\operatorname{dim}_{F_{0}}(U)=$ $\aleph_{0}$. Let $V=U \otimes_{F_{0}} F$, but endow it only with the structure preserved by the semilinear automorphisms. Let $\sim$ be the equivalence relation on $V-(0)$ defined by $v \sim v^{\prime}$ iff $v^{\prime}=\alpha v$ for some $\alpha \in F$. By effecting a set theoretic manipulation, we can assume $V / \sim=P^{*}$. Note that for $u, u^{\prime} \in U, u \sim u^{\prime}$ iff 
$u^{\prime}=\alpha u$ for some $\alpha \in F_{0}$. More generally, it is easy to verify: If $u_{1}, \ldots, u_{k} \in U$ are linearly independent and $\sum \alpha_{i} u_{i} \in U$, then each $\alpha_{i} \in F_{0}$.

Let $Q=U / \sim=\{u / \sim: u \in U\}$. So (*) implies (a). Every automorphism of $Q^{*}$ extends to an automorphism of $U$, and every automorphism of $U$ extends uniquely to a linear automorphism of $V$, hence to an automorphism of $P^{*}$. This proves (b). (c) is immediate. To prove $(\mathrm{d})$, assume $\operatorname{tp}(\bar{a})=$ $\operatorname{tp}(\bar{b})$. Let $\sigma$ be an automorphism of $P$ with $\sigma(\bar{a})=\bar{b}$. Without loss of generality $a_{1}, \ldots, a_{k}$ are independent and $a_{i}$ depends on $a_{1}, \ldots, a_{k}$ for $i \geq$ $k$. Choose $u_{i} \in U$ such that $a_{i}=u_{i} / \sim$. So $u_{i}=\sum_{j \leq k} \alpha_{i, j} u_{j}$ for some matrix $\left(\alpha_{i, j}\right)$ from $F$. Extend $\sigma$ to a semilinear automorphism $\bar{\sigma}$ of $V$. Since each $\alpha_{i, j}$ is in the prime field (by $\left.(*)\right), \bar{\sigma}\left(u_{i}\right)=\sum_{j \leq k} \alpha_{i, j} \bar{\sigma}\left(u_{j}\right)$. But clearly $\operatorname{stp}\left(u_{1}, \ldots, u_{k}\right)=\operatorname{stp}\left(\bar{\sigma} u_{1}, \ldots, \bar{\sigma} u_{k}\right) ; \operatorname{so~} \operatorname{stp}\left(u_{1}, \ldots, u_{n}\right)=\operatorname{stp}\left(\bar{\sigma} u_{1}, \ldots, \bar{\sigma} u_{n}\right)$, and $\operatorname{stp}(\bar{a})=\operatorname{stp}(\bar{b})$. (The strong types are evaluated in $V$, but for elements of $P$ it is the same as taking them in $P$.)

Let $h_{b}: P_{b} \rightarrow P^{*}$ be an isomorphism, and let $Q_{b}$ the inverse image of $Q^{*}$ under $h_{b}$. Let $N_{0}=M \cup \dot{\cup} Q^{*}$ (model-theoretic disjoint union) and let $N$ be $N_{0}$ enriched by the relation $H:(b, x, y) \in H$ iff $b \in D_{3}, x \in P_{b}, y \in Q^{*}$ and $y=h_{b}(x)$.

Lemma 3.12. For every finite $X \subset N$ there exists a finite $Y \subset N_{0}$ such that every permutation of $\hat{D} \cup Q^{*}$ that extends to an automorphism of $N_{0}$ fixing $Y$, extends to an automorphism of $N$ fixing $X$. For $X=\varnothing$ one may choose $Y=\varnothing$.

Proof. Let $X=X_{M} \cup X_{P^{*}}, X_{M} \subset M, X_{P^{*}} \subset P^{*}$. Let $E=\bigcup_{b \in D_{3}} Q_{b}$. Let $X^{\prime}$ be a finite subset of $\hat{D} \cup E$ such that $\operatorname{tp}\left(\bar{X} / X^{\prime}\right) \vdash \operatorname{tp}\left(\bar{X} / \hat{D} \cup E\right.$ ) in $N_{0}$ (where $\bar{X}$ is an enumeration of $X$; if $X=\varnothing$, choose $\left.X^{\prime}=\varnothing\right)$. Let

$$
Y=\left(X^{\prime} \cap(\hat{D})\right) \cup\left\{b \in D_{3}: P_{b} \cap X^{\prime} \neq \varnothing\right\} \cup\left\{h_{b}(x): b \in D_{3}, x \in Q_{b} \cap X^{\prime}\right\} .
$$

Let $\sigma$ be a permutation of $\hat{D} \cup P^{*}$ that extends to an automorphism of $N_{0}$ fixing $Y$. As the $P_{b}$ 's may be assumed to be pairwise disjoint, there exists a unique permutation $\tau$ of $\hat{D} \cup P^{*} \cup E$ such that $\sigma \subset \tau$ and $h_{\tau b}(\tau x)=\tau\left(h_{b}(x)\right)$ for any $b \in D_{3}$ and any $x \in Q_{b}$. Note that $\tau$ fixes $X^{\prime}$ pointwise.

Claim. $\tau$ is a partial automorphism of $N_{0}$.

Proof. Let $\bar{\sigma}$ be an automorphism of $N_{0}$ extending $\sigma$. Let $\rho=\bar{\sigma}^{-1} \tau$. It suffices to show that $\rho$ is a partial automorphism of $N_{0}$. Now $\rho$ is the identity on $\hat{D} \cup P^{*}$. Since $N_{0}=M \cup \dot{\cup} P^{*}$, the only question is whether $\rho$ is elementary on $\hat{D} \cup E$. In other words, for any $n$ and any distinct $b_{1}, \ldots, b_{n} \in D_{3}$, one must show that $\rho \mid\left(\hat{D} \cup Q_{b_{1}} \cup \cdots \cup Q_{b_{n}}\right)$ is elementary. Proceeding by induction on $n$, we may assume that $\rho$ fixes $G=\hat{D} \cup \bigcup_{i<n} Q_{b_{i}}$ pointwise. Since $Q_{b}$ has either an $F_{0}$-projective space structure respected by $\rho$, and no more, or else no 
structure at all, it is clear at least that $\rho \mid Q_{b} \cup\left\{b_{n}\right\}$ is a partial automorphism. To prove that $\rho \mid\left(\hat{D} \cup P_{b_{1}} \cup \cdots \cup P_{b_{n}}\right)$ is elementary thus amounts to this: if $\bar{c}, \bar{d}$ are tuples from $Q_{b_{n}}$ realizing the same type over $b_{n}$, then they realize the same type over $G$. By 3.9 and 3.10, $P_{b_{n}} \perp G$. Thus $\bar{c} \perp G \mid\left\{b_{n}\right\}$ and $\bar{d} \perp G \mid\left\{b_{n}\right\}$. By $3.11(\mathrm{~d}), \operatorname{stp}\left(\bar{c} / b_{n}\right)=\operatorname{stp}\left(\bar{d} / b_{n}\right)$. So $\operatorname{tp}\left(\bar{c} / b_{n}\right)=\operatorname{tp}\left(\bar{d} / b_{n}\right)$, and the proof of the claim is finished.

Let $\tau_{0}$ be an automorphism of $N_{0}$ extending $\tau$. Since $\tau$ fixes $X^{\prime}$ pointwise, so does $\tau_{0} ;$ so $\operatorname{tp}(\bar{X} / \hat{D} \cup E)=\operatorname{tp}\left(\tau_{0} \bar{X} / \hat{D} \cup E\right)$. Let $\rho_{0}$ be an automorphism of $N_{0}$ fixing $\hat{D} \cup E \cup P^{*}$ and sending $\bar{X}$ to $\tau_{0} \bar{X}$, and let $\bar{\tau}=\tau_{0} \rho_{0}^{-1}$. Then $\bar{\tau}$ extends $\tau$, fixes $X$ pointwise, and is an automorphism of $N_{0}$. Moreover, $\bar{\tau}$ preserves $H$, hence is an automorphism of $N$.

Remark 3.13. The proof of Lemma 3.12 used only the following information about $N$ : it is obtained from $M$ by freely adjoining the structure $P^{*}$, and then adding bijections $h_{b}$ from a subset of $P_{b}$ onto $Q^{*}$, such that each $h_{b}$ extends to an isomorphism of $P$ with $P^{*}\left(b \in D_{3}\right)$. This remains true of a saturated extension $\tilde{N}$ of $N$. Hence the lemma is true of such a saturated extension. Therefore, the lemma implies that $\hat{D} \cup Q^{*}$ is $\aleph_{0}$-stable, $\aleph_{0}$-categorical as a definable subset of $N$, and in fact $Q^{*}$ is strictly minimal as a definable subset of $N$.

We can now finish the proof of the theorem. Each $P_{b}$ is uniformly algebraic over $Q_{b}$, so $\left(\bigcup_{b \in D_{3}} P_{b}\right)$ is $\left(\bigcup_{b \in D_{3}} Q_{b}\right)$-analyzable in $N$. Since the $Q_{b}$ 's are the classes of a definable equivalence relation, and each is definable isomorphic to $Q^{*}$ (via $\left.h_{b}\right),\left(\bigcup_{b \in D_{3}} P_{b}\right)$ is $\hat{D} \cup Q^{*}$-analyzable in $N$. Thus $\hat{D} \cup\left(\bigcup_{b \in D_{3}} P_{b}\right)$ is $\hat{D} \cup Q^{*}$-analyzable. Using the last remark, Lemma 3.6 shows that $\hat{D} \cup\left(\bigcup_{b \in D_{3}} P_{b}\right)$ is $\aleph_{0}$-stable, $\aleph_{0}$-categorical as a definable subset of $N$. But by $3.10, M$ is $\hat{D} \cup\left(\bigcup_{b \in D_{3}} P_{b}\right)$-analyzable. Hence so is $N$, and another use of Lemma 3.6 proves that $N$ itself is $\aleph_{0}$-stable, $\aleph_{0}$-categorical. Now by $3.3(\mathrm{a}), N$ is $\hat{D} \cup Q^{*}$ analyzable.

Taking $X=\varnothing$ in Lemma 3.12, we see that the structure induced on $\hat{D}$ in $N$ is the same as the structure induced on it as a definable subset of $M$. In particular the Morley rank, multiplicity of $\hat{D}$ in $N$ is less than $k, m$. But the lemma also says that $P^{*}$ is strongly minimal in $N$; so if $k>1$ then

$$
(e-\operatorname{rk}(N), e-\operatorname{mult}(N)) \leq\left(\operatorname{rk}\left(\hat{D} \cup P^{*}\right), \operatorname{mult}\left(\hat{D} \cup P^{*}\right)\right)<(k, m) .
$$

We are thus done unless $k=1$. (Strictly speaking, $M$ is not a reduct of $N$ because of $Q^{*}$; but the distinction is clearly only poetic.)

We may therefore assume $e-\operatorname{rk}(M)=1$. So $M$ is $P_{1} \cup \cdots \cup P_{n}$-analyzable, where each $P_{i}$ is strictly minimal and 0 -definable, and the $P_{i}$ 's are pairwise orthogonal. The reduction of the essential multiplicity $n$ is effected in the same way as that of the rank was above. Each projective $P_{i}$ is first replaced with a $Q_{i}$ provided by 3.11 . Then, if two distinct $P_{i}$ 's (say $P_{1}$ and $P_{2}$ ) are over fields of the same characteristic, or both disintegrated, one adds an arbitrary 
isomorphism $h$ between $P_{1}$ and $P_{2}$. Let $\widetilde{M}=(M, h)$. Then one sees as above the $\widetilde{M}$ is $\aleph_{0}$-stable, $\aleph_{0}$-categorical, has essential rank 1 , and essential multiplicity $n-1$. After finitely many steps, all the projective $P_{i}$ 's become over distinct prime fields, and there remains at most one disintegrated $P_{i}$ (say $\left.P_{0}\right)$. If one wishes, one may further swallow $P_{0}$ into $P_{1}$ (if $n \geq 1$ ). This finishes the proof.

Remark 3.14. The introduction of projective spaces over prime fields is unavoidable. Suppose for example that one is presented with a model $M$, an infinite definable set $D$ in $M$, and for $a \in D$ a vector space $V_{a}$ over a finite field $F_{a}$. The $F_{a}$ 's are abstractly isomorphic of course, but there may be no $M$-definable uniform system of isomorphisms between the $F_{a}$ 's. Suppose, however, that $M$ is interpretable in a finite-dimensional $\widetilde{M} \in \mathbf{C}$. Let $D_{0} \subset D$ be the locus of a complete stationary nonalgebraic type of $\widetilde{M}$. For $a \in D_{0}$, let $U_{a}$ be a minimal infinite $\widetilde{M}$-definable subgroup of $V_{a}$, and let $P_{a}$ be the associated projective space. The $P_{a}$ 's must be pairwise nonorthogonal. So for $a, a^{\prime} \in D_{0}$ there exists a unique 0 -definable bijection between $P_{a}$ and $P_{a^{\prime}}$. This map induces an isomorphism of $\widetilde{F}_{a}$ and $\widetilde{F}_{a^{\prime}}$, where $\widetilde{F}_{a}$ is the underlying field of $U_{a}$ (and $P_{a}$ ). The uniqueness shows that one has a commuting system of field isomorphisms. Thus if one wants to find an expansion in which no vector spaces over smaller fields are introduced, i.e. $F_{a}=\widetilde{F}_{a}$ for each $a$, then one must first find an expansion in which the $F_{a}$ 's become identified with one $F$. The following example shows that this may not be possible.

Example 3.15 (Cherlin). Let $W$ be a vector space over a finite field $K$ with $l+1$ elements, $l>1$. Let $F_{0}$ be any prime field, and let $F$ be the extension of degree $l$. Let $f(x)$ be an irreducible polynomial over $F_{0}$ with exactly $l$ solutions in $F$. Let $P$ be the projective space associated with $W$; each $p \in P$ will literally be considered as a 1-dimensional subspace of $W$ without the 0 point. For $b \in P$, let $F_{b}$ be a copy of $F$, so that the set-theoretic intersection of $F_{b}$ and $F_{b^{\prime}}$ is just $F_{0}$ for $b \neq b^{\prime}$. Let $G_{b}$ be the group of automorphisms of $F_{b}$ over $F_{0}$, and let $X_{b}$ be the set of roots of $f(x)$ in $F_{b}$. Then $\left(G_{b}, X_{b}\right)$ is a cyclic group of order $l$ acting faithfully on a set of order $l$. So $\left(G_{b}, X_{b}\right) \approx\left(K^{x}, b\right)$. let $h_{b}: b \rightarrow X_{b}, G_{b} \rightarrow K^{x}$ be an isomorphism.

Suppose $\widetilde{M}$ is a structure containing $W, P, F_{b}, X_{b}, G_{b}, h_{b}(b \in P)$, and such that the $F_{b}$ 's are uniformly isomorphic to $F$ for each $b$ in some infinite subspace $P_{0}$ of $P$. Let $W_{0}=\{0\} \cup \cup P_{0}$. Let $\alpha$ be a solution of $f(x)$ in $F$. For each the isomorphism of $F_{b}$ and $F$ takes $\alpha$ to some element $\alpha_{b}$ of $X_{b}$. $h_{b}$ takes it back to a point in $b$. So one has a definable set $Y$ that intersects each 1-dimensional subspace of $W_{0}$ in a unique point. It follows that $\widetilde{M}$ is unstable. For if $\widetilde{M}$ were stable, let $r$ be the generic type of the connected component of $W_{0}$. For $a \vDash r, \alpha a \in Y$ for a unique $\alpha \in K-(0)$. But if $a \vDash r$ then $\beta a \vDash r$ for $\beta \in K-(0)$, so $\alpha \beta a \in Y$, so $\alpha \beta=\alpha$. This proves that $K$ is the 2-element field, contrary to assumption. 
Hence if one further adds, for each $b$, a vector space $V_{b}$ over $F_{b}$, then by 3.14 there is no finite dimensional $\aleph_{0}$-categorical, $\aleph_{0}$-stable expansion in which the $V_{b}$ 's remain strongly minimal.

Remark 3.16. If we start with $M \in \mathbf{C}_{m}$ (the class of structures of modular type studied in [C]), the theorem gives an interpretation of $M$ in an almost strongly minimal structure.

Example 3.17. It is not possible to embed an arbitrary totally categorical structure in an almost strongly minimal structure. The reason is that once one has a group structure, by [PH] almost no further structure can be added to it. For let $A=(Z / 4 Z)^{(\omega)}$. Note that if $B$ is a subgroup of $A$ and $B \cap 2 A=(0)$ then $B=(0)$. Moreover, if $B \cap 2 A$ is finite then so is $B$ (and $\operatorname{card}(B) \leq$ $\left.\operatorname{card}(B \cap 2 A)^{2}\right)$. Suppose $A$ sits inside an almost strongly minimal theory. Say $A \subset \operatorname{acl}\left(D_{1} \cup \cdots \cup D_{n}\right)$, where the $D_{i}$ 's are strongly minimal. After absorbing some parameters, we may assume that a generic $a \in A$ is equialgebraic with some independent $d_{1}, \ldots, d_{m}$ in $D=D_{1} \cup \cdots \cup D_{n}$. Say $d_{1}, \ldots, d_{k}$ is a maximal subset of $d_{1}, \ldots, d_{m}$ independent from $2 a$. Consider $N=\left\{x \in A: \operatorname{stp}\left(x / d_{1} \cdots d_{k}\right)=\operatorname{stp}\left(a / d_{1} \cdots d_{k}\right)\right\}$. For each generic $c \in 2 a$ there are finitely many (but at least one) $x \in N$ such that $2 x=c$. By [PH], the stabilizer $S$ of $N$ is infinite. By the remark made at the beginning, $S \cap 2 A$ is infinite. If follows that for $x \in N$ there exist infinitely many $y \in N$ with $2 x=2 y$. This is a contradiction.

\section{STRUCTURES OF DISINTEGRATED TYPE}

The main result of this section is the classification of a certain class of totally categorical structures of disintegrated type. Every totally categorical structure can be expanded to a member of this class by the naming of finitely many constants. This gives an explicit proof of the original Ahlbrandt-Ziegler result for the disintegrated case, without using combinatorial theorems.

Let $\mathbf{D}$ be the class of totally categorical structures with a distinguished 0definable set $D$ of disintegrated type.

We also investigate the situation before constants have been added. We show that the information that is lost by the addition of constants is controlled by nilpotent automorphism groups. (The proof gives more detailed information.)

Weaker versions of the results hold for the class $\mathbf{C}_{1}=\{M \in \mathbf{C}: M \subseteq \operatorname{acl}(D)$ for some 0 -definable, modular, strictly minimal set $D\}$. This is not our main interest here, so we only indicate them in passing.

We start by defining an operation $M^{*} F$; starting with $M \in \mathrm{C}_{1}, M^{*} F$ gives another structure in $\mathbf{C}_{1}$, obtained by freely joining copies of the finite structure $F$ along a Grassmannian associated with $M$.

Notation. A definable substructure $E$ of a finite model $F$ is a definable subset of $F$, considered as a structure in its own right, in such a way that every automorphism of $E$ extends to an automorphism of $F$. 
If $D$ is a strictly minimal set, let $[D]^{n}=\{w \subset D: w$ is algebraically closed, of dimension $n$ \}.

If $M \in \mathbf{C}_{1}$, let $D$ be a strictly minimal, modular, 0-definable subset of $M$, and let $M(w)=\operatorname{acl}(w) \cap M$ for $w \in[D]^{n}$. Let $M[n]=\bigcup\left\{M(w): w \in[D]^{n}\right\}$. Note that $M[n]$ does not depend on the choice of $D$. Let $E_{n}(M)$ be a finite structure isomorphic to $M(w)$ for $w \in[D]^{n}$.

Definition 4.1. We now define $M^{* n} F$. The data are a model $M \in \mathrm{C}_{1}$, a finite structure $F$, and a definable substructure $E$ of $F$ isomorphic to $E_{n}(M)$. ( $E$ is considered to be given as a part of the presentation of $F . n$ can be recovered from $E$, so we will sometimes write $M^{*} F$ for $M^{* n} F$.) For $w \in[D]^{n}$, let $(F(w), E(w))$ be a copy of $(F, E)$. Choose the copies so that $E(w)=M(w)$ for each $w \in[D]^{n}$, but otherwise there is no intersection: $\{M\} \cup\left\{F(w)-E(w): w \in[D]^{n}\right\}$ is a pairwise disjoint family. let $M^{*} F$ be the model with universe $M \cup \bigcup\left\{F(w): w \in[D]^{n}\right\}$, and whose structure consists of the structure on $M$ together with the relations $\left\{(w, \bar{x}): w \in[D]^{n}, \bar{x} \in F(w)\right.$, $F(w) \vDash R(\bar{x})\}$, where $R$ is a definable relation of $F$. It is clear that every automorphism of $M$ extends to one of $M^{*} F$. It follows that $M^{*} F \in \mathrm{C}_{1}$.

We will be interested in structures of the form $D^{* 0} F_{0}{ }^{* 1} F_{1}{ }^{* 2} \ldots{ }^{* n} F_{n}$, where $D$ is a pure set. It is therefore useful to have an explicit criterion for recognizing iterations of the operation *

Definition 4.2. Let $M$ be a structure, $D$ a projective space or a set, and $M(w)$ a substructure of $M$ for $w \in[D]^{\leq k}$. We say that $\left(M(w): w \in[D]^{\leq k}\right)$ is in free amalgamation within $M$ if

(i) $M(w) \cap M\left(w^{\prime}\right)=M\left(w \cap w^{\prime}\right)$ for $w, w^{\prime} \in[D]^{\leq k}$.

(ii) If $\sigma$ is a permutation of $D \cup\left(\bigcup_{w}, M(w)\right)$ that leaves $D$ invariant and carries $M(w)$ isomorphically onto $M(\sigma w)$ for $w \in[D]^{\leq k}$, then $\sigma \mid\left(\bigcup_{w} M(w)\right)$ is a partial automorphism of $M$.

Criterion 4.3. Let $M \in \mathbf{C}_{1}$ and let $D$ be a 0 -definable, modular, strictly minimal set in $M$. Then $M$ is isomorphic to $D^{*} F_{0}{ }^{*} \ldots{ }^{*} F_{n}$ for some $F_{0}, \ldots, F_{n}$ iff $M=M[n]$, and $\left(M(w): w \in[D]^{\leq n}\right)$ is in free amalgamation within $M$. (The notion of isomorphism is for permutational structures.)

Theorem 4.4. Every totally categorical $M \in \mathbf{D}$ has an expansion by constants isomorphic to $D^{* 0} F_{0}{ }^{* 1} F_{1}{ }^{*} \ldots{ }^{* n} F_{n}$ for some $F_{1}, \ldots, F_{n}$ and some disintegrated $D$. (Both structures are given their canonical languages.)

Remark 4.5. $F_{0}$ and $F_{1}$ can be chosen to be the trivial (0- and 1-element) structures. But if this requirement is not made, then the constants in the statement of the theorem can be chosen from $D$. In fact, the proof will show that it suffices to name an element of the Grassmannian $[D]^{k}$ for some $k$.

Corollary 4.6. If $H$ is a definable substructure of $F_{n}$, define $D^{* 0} F_{0}{ }^{* 1} F_{1} \cdots$ ${ }^{* n} F_{n} \mid H$ as follows: let $H(w)$ be the substructure of $F_{n}(w)$ corresponding to $H$, 
in a given copy $F_{n}(w)$ of $F_{n}$ in $D^{* 0} F_{0}{ }^{* 1} F_{1} \cdot{ }^{* n} F_{n}$. Let $D^{* 0} F_{0}{ }^{* 1} F_{1} \cdot{ }^{* n} F_{n} \mid H$ $={ }_{\mathrm{df}} \bigcup_{w \in[D]^{n}} H(w)$. Then every totally categorical structure $M \in \mathbf{D}$ has the form $M=D^{* 0} F_{0}{ }^{* 1} F_{1} \cdot{ }^{* n} F_{n} \mid H$. This provides a finite code for $M$.

Proof. Let $D$ be a strictly minimal, 0-definable subset of $M$. Let $F$ be a finite subset of $D$ such that $N=(D, F)$ has the form $D^{* 0} F_{0}{ }^{* 1} F_{1} \cdots{ }^{* n} F_{n}$. Let $M^{\prime}=\operatorname{acl}_{M}(D-F)$. Then $M^{\prime}$ is a definable subset of $N$, so it has the required form. But $M^{\prime}$ is an elementary submodel of $M$, so $M^{\prime} \approx M$, and hence $M$ has the correct form also.

The code for $N$ is a finite system $\left(M(w): w \in[n]^{\leq n}\right)$ of finite structures in free amalgamation, with the further requirement that $M(n)$ contain $n$ as a definable, disintegrated substructure. Note that any such code can be written uniquely as $n^{* 0} F_{0}{ }^{* 1} F_{1}{ }^{*} \cdots^{* n} F_{n}$, with all $F_{i}$ 's finite; and it is the code of $\omega^{* 0} F_{0}{ }^{*} \ldots{ }^{* n} F^{n}$. The code for $M$ is the code for $N$, together with $H$. (Admittedly this is not entirely satisfactory, which is why the section does not end here.)

Proof of Theorem 4.4. Let $D$ be a 0-definable strictly minimal set of $M$. Let $n$ be the Morley rank of $M$. For $w \in[D]^{<\omega}$, let $M(w)=\operatorname{acl}(w) \cap M$. So $M=\bigcup\left\{M(w): w \in[D]^{n}\right\}$. For $w \in[D]^{m}$ (with $m \geq n$ ), $M(w)=$ $\bigcup\left\{M\left(w^{\prime}\right): w^{\prime} \in[w]^{\leq n}\right\}$. For $w \in[D]^{k}, k \leq n$, and $S \subset D$, let

$$
G(w, S)=\operatorname{Aut}\left(M(w) / \bigcup\left\{M\left(w^{\prime} \cup S\right): w^{\prime} \in[w]^{<k}\right\}\right) .
$$

Note that for $S$ disjoint from $w, G(w, S)$ depends only on $\operatorname{card}(S)$; and as card $(S)$ grows larger, $G(w, S)$ becomes a smaller subgroup of $\operatorname{Aut}(M(w))$. Thus there is some $d(k)$ such that $G(w, S)$ is as small for $\operatorname{card}(S)=d(k)$ as it ever is. Let $d=\max (d(k): k \leq n)$, and let $F$ be a subset of $D$ of cardinality $d$. I claim that the system $\left(M(w \cup F): w \in[D-F]^{\leq n}\right\}$ is in free amalgamation. By Criterion 4.3 this implies that $M$, enriched with a constant for each element of $F$, is of the required form.

We may assume inductively that $\left(M(w \cup F): w \in[D-F]^{<k}\right)$ is free, and prove the same for $\left(M(w \cup F): w \in[D-F]^{\leq k}\right)$. Note that (i) is immediate. To prove (ii), let $\sigma$ be a permutation of $\bigcup\left\{M(w \cup F): w \in[D-F]^{\leq k}\right\}$ that leaves $D-F$ invariant and carries $M(w \cup F)$ isomorphically onto $M(\sigma w \cup F)$ for $w \in[D]^{\leq k}$ (isomorphically means over $F$ now). By induction,

$$
\sigma \mid\left(\bigcup\left\{M(w \cup F): w \in[D-F]^{<k}\right\}\right)
$$

is a partial automorphism of $M$ over $F$. If $k>1$ then $D \subseteq(\bigcup\{M(w \cup F)$ : $\left.\left.w \in[D-F]^{<k}\right\}\right)$. If $k=1$, then trivially $\sigma \mid D \cup M(F)$ is a partial automorphism of $M$. Correcting $\sigma$ by an automorphism of $M$, we may thus assume that $\sigma$ acts as the identity on $\bigcup\left\{M(w \cup F): w \in[D-F]^{<k}\right\} \cup D$. In particular, $\sigma$ leaves each $M(w)$ invariant, and $\sigma \mid M(w) \in G(w, F)$ for each $w$. By the choice of $d, \sigma \mid M(w) \in G(w, J)$ for every finite $J \supset F$ with $w \cap J=\varnothing$. Let $\sigma_{w}^{*}=(\sigma \mid M(w)) \cup\left(\right.$ the identity on $\left.\bigcup\left\{M\left(w^{\prime}\right): w^{\prime} \in[D]^{n}, w \nsubseteq w^{\prime}\right\}\right) . \sigma^{*}$ is a 
permutation of $M$ (fixing $D$ ); I claim that it is an automorphism. It suffices to show that $\sigma \mid M(w)$ is a partial automorphism over $\bigcup\left\{M\left(w^{\prime}\right): w^{\prime} \in[J]^{\leq n}, w \nsubseteq\right.$ $\left.w^{\prime}\right\}$ for each finite $J$ with $F \subset J \subset D$. But this is just the meaning of the fact that $\sigma \mid M(w) \in G(w, J-w)$. Thus $\sigma_{w}^{*}$ is a partial automorphism of $M$. Let $\sigma_{w}^{*}=\sigma_{u}^{*} \mid \cup\left\{M\left(w^{\prime} \cup F\right): w^{\prime} \in[D-F]^{\leq k}\right\}$. Each $\sigma_{w}^{*}$ agrees with $\sigma$ on $M(w)$ and fixes pointwise every other $M\left(w^{\prime}\right)$ with $w^{\prime} \in[D]^{k}$, as well as $\bigcup\left\{M(w \cup F): w \in[D-F]^{<k}\right\}$. So the product of all the $\sigma^{\#}(w)\left(w \in[D-F]^{k}\right)$ converges to $\sigma$. Thus $\sigma$ is a partial automorphism of $M$. This finishes the proof.

Example 4.7a. Let $A$ be a finite Abelian group of exponent $e$. Let $D$ be an infinite set and $k$ an integer. Let $W=[D]^{k}$. Let $N$ be the structure $(D, W, A \times W, R)$ where $\left(\left(a^{1}, w^{1}\right), \ldots,\left(a^{j}, w^{j}\right)\right) \in R$ iff $a^{1}+\cdots+a^{j}=0$, and $\left(w^{i}: i \leq j\right)$ is an enumeration of the set of all $k$-element subsets of some $k+e-1$-element subset of $D$. Note that for any map $c:[D]^{k-1} \rightarrow A$, the map $(a, w) \rightarrow\left(a+\sum\left\{c\left(w^{\prime}\right): w^{\prime} \in[w]^{k-1}\right\}, w\right)$ is an automorphism of $N$. (This example is akin to examples by Cherlin and Lachlan presented in [C].)

Example 4.7b (twisted version). This example shows that the exponent of the group cannot be computed from the dimension of the Grassmannian and the location of the link. Let $A$ be a finite Abelian group of arbitrary exponent. Let $D, W, k$ be as above. Given $w \in[D]^{k}$, let $X_{w}$ be a set of the same cardinality as $A$. Given an enumeration $\bar{w}$ of $w$, let $h_{\bar{w}}: A \rightarrow \operatorname{Sym}\left(X_{w}\right)$ be a regular action of $A$ on $X_{w}$. Arrange that if $\varphi \in \operatorname{Sym}(w), \bar{w}^{\prime}=\varphi(\bar{w})$, then for all $a \in A, h_{\bar{w}^{\prime}}(a)=h_{\bar{w}}\left((-1)^{\operatorname{sign}(\varphi)+1} a\right)$. Choose the $X_{w}$ 's disjoint. So specifying an automorphism over $D$ of $M_{0}=D \cup \bigcup_{w} X_{w}$ with the structure described so far is the same as giving a function $\alpha: D^{k} \rightarrow A$ such that $\alpha(\varphi \bar{w})=$ $(-1)^{\operatorname{sign}(\varphi)+1} \alpha(\bar{w})$ for any $\bar{w} \in D^{k}$ and any $\varphi \in \operatorname{Sym}\left(\left\{w_{1}, \ldots, w_{k}\right\}\right)$. Let $G=$ $\left\{\alpha\right.$ : for all $t=\left\{d_{0}, \ldots, d_{k}\right\} \in[D]^{k+1}$, letting $t^{i}=\left(d_{0}, \ldots, d_{i-1}, d_{i+1}, \ldots, d_{k}\right)$, one has $\left.\sum_{0 \leq i \leq k}(-1)^{i} \alpha\left(t^{i}\right)=0\right\}$. There exists a unique expansion $M$ of $M_{0}$ such that $\operatorname{Aut}_{M}(D)=\operatorname{Sym}(D)$ and $\operatorname{Aut}(M / D)=G$. Automorphisms of $M$ can be obtained as follows: let $<$ be a linear ordering of $D$. Let $c:[D]^{k-1} \rightarrow A$ be any $k-1$ coloring of $D$; identify $[D]^{k-1}$ with the increasing $k-1$-tuples. For increasing $w \in[D]^{k}$, let $\alpha(\bar{w})=\sum_{i}(-1)^{i} c\left(w_{1}, \ldots, \hat{w}_{i}, \ldots, w_{n}\right)$; and extend $\alpha$ to $[D]^{k}$ in the obvious way. Then it is easy to check that $\alpha \in G$.

Proposition 4.8. Let $M \in \mathbf{D}_{1}$. After adding finitely many imaginary sorts to $M$ from $M^{\mathrm{eq}}, M$ can be analyzed as follows. Let $D$ be strictly minimal and 0-definable, $M_{i}=\bigcup\left\{M(w): w \in[D]^{i}\right\}$. Then for each $i \geq 1$ there exists $a$ 0-definable set $M_{i, 0}$ such that $M_{i-1} \subseteq M_{i, 0} \subseteq M_{i}$, and

(i) $M_{0}$ is finite.

(ii) $M_{1,0}=M_{0} \cup D$. For $i \geq 2, \operatorname{Aut}\left(M_{i, 0} / M_{i-1}\right)$ is nilpotent of class $\leq i$.

(iii) $M_{i}=M_{i, 0}{ }^{* i} F$ for some finite $F(i \geq 1)$. 
The reader is warned that aside from the bound on the nilpotency class in (ii), the proposition has an entirely trivial proof. The proof below is given not in order to get the bound, but rather as it gives a model theoretic meaning to the nilpotency. The trivial proof is given at the end of the chapter.

Proof. Let $R=\operatorname{rk}(M)$. Let $M^{*}$ consist of finitely many sorts from $M^{\text {eq }}$, all of rank $\leq R$, and enough so that:

(*) For every $r \leq R$ and every $w \in[D]^{r}$, letting $M^{*}(w)=\operatorname{acl}(w) \cap M^{*}$, the correspondence between definitionally closed subsets of $M^{*}(w)$ and subgroups of $\operatorname{Aut}\left(M^{*}(w)\right)$ given by $X \rightarrow \operatorname{Aut}\left(M^{*}(w) / X\right), H \rightarrow\left\{e \in M^{*}(w)\right.$ : he =e for all $h \in H\}$ is a 1-1 correspondence. WLOG $M^{*}=M$, so (\#) holds for $M(w)=\operatorname{acl}(w) \cap M$. For $x \subset w \subset F \subset D$ with $\operatorname{card}(x)=k, \operatorname{card}(w)=r$, $\operatorname{card}(F)=n$, define

$G(w)=\operatorname{Aut}(M(w))$.

$G(x ; w)=\operatorname{Aut}\left(M(w) / \bigcup\left\{M\left(w^{\prime}\right): w^{\prime} \in[D]^{r}, x \subseteq w^{\prime}, w \neq w^{\prime}\right\}\right)$.

$G(k ; w)=\bigcap\left\{G(x ; w): x \in[w]^{k}\right\}$,

$M(x ; w)=\{e \in M(w): e$ is fixed by $G(x ; w)\}$.

$M(k ; w)=\bigcup\{M(x ; w): x \subset w, \operatorname{card}(x)=k\}$.

$M_{r, k}=\bigcup\left\{M(k ; w): w \in[D]^{r}\right\}$.

$G(x, w, n)=\operatorname{Aut}\left(M(w) / \bigcup\left\{M\left(w^{\prime}\right): x \subset w^{\prime} \subset F, \operatorname{card}\left(w^{\prime}\right)=r, w^{\prime} \neq w\right\}\right)$.

(It does not depend on $F$.)

$G(k, w, n)=\bigcap\{G(x, w, n): x \subset w, \operatorname{card}(x)=k\}$.

Clearly $G(x, w, n)=G(x ; w)$ for large enough $n$.

Claims. (a) $M_{r}=M_{r, 0}{ }^{* r} F$ for some $F$.

(b) $\operatorname{dcl}\left(M_{r, r-1}\right)=\operatorname{dcl}\left(M_{r-1}\right)$ for $r \geq 2 . \operatorname{dcl}\left(M_{1,0}\right)=\operatorname{dcl}\left(M_{0} \cup D\right)$.

(c) Let $F \subset D$ be finite, $w \in[D]^{r}$. Then

$$
\operatorname{Aut}\left(M(w) / \bigcup\left\{M\left(w^{\prime}\right): w^{\prime} \in[F]^{\leq r}\right\}\right)=\operatorname{Aut}(M(w) / M(w \cap F)) .
$$

(d) $[G(r-1 ; w), G(k+1 ; w)] \subseteq G(k, w) \quad\left(k+1 \leq r-1, w \in[D]^{r}\right)$.

Proofs. (a) Let $(F, E)$ be a finite structure isomorphic to $(M(w), M(0 ; w))$ for $w \in[D]^{r} . \quad M_{r}=M_{r, 0} \cup \bigcup\left\{M(w): w \in[D]^{r}\right\}$. To show that $M_{r}=M_{r, 0}^{*} F$ we need to show that (i) $\left\{M_{r, 0}\right\} \cup\left\{M(w)-M(0 ; w): w \in[D]^{r}\right\}$ is pairwise disjoint, and (ii) every permutation $\sigma$ of $M_{r}$ such that $\sigma \mid M_{r, 0}$ is an automorphism and $\sigma$ takes $M(w)$ isomorphically to $M(\sigma w)$ for each $w$, is an isomorphism of $M_{r}$.

(i) is clear: if $e \in(M(w)-M(0 ; w)) \cap M\left(w^{\prime}\right)$ with $w^{\prime} \neq w$ then for every $\sigma \in G(0 ; w), \sigma(e)=e$ (else $\sigma$ could not extend to an automorphism fixing $\left.M\left(w^{\prime}\right)\right)$; thus $e \in M(0 ; w)$, a contradiction. (ii) is similar to the end of the proof of 4.4. [By correcting with an automorphism of $M_{r}$ extending $\sigma \mid M_{r, 0}$ we may assume $\sigma$ fixes $M_{r, 0}$. So $\sigma \mid M(w)$ is an automorphism of $M(w)$. Since $\sigma$ fixes $M(0 ; w), \sigma \mid M(w) \in G(0 ; w)$. So $\sigma_{u}^{\#}=(\sigma \mid M(w)) \cup$ $\left(\bigcup\left\{\operatorname{id} \mid M\left(w^{\prime}\right): w^{\prime} \in[D]^{r}, w^{\prime} \neq w\right\}\right)$ is an automorphism of $M_{r}$, for each $w$. Thus so is $\sigma=\prod\left\{\sigma_{w}^{\#}: w \in[D]^{r}\right\}$.] 
(b) We will show that $M(x ; w)=M(w) \cap \operatorname{dcl}(M(x) \cup w)$ when $x \subset w$ and $\operatorname{card}(x)=r-1, \operatorname{card}(w)=r$. If $\sigma$ is an automorphism of $M$ fixing $w$ and $Y=\bigcup\{\operatorname{acl}(x \cup\{p\}): p \in D-w\}$, then $\sigma$ leaves $M(w)$ invariant and $\sigma \mid M(w) \in$ $G(x ; w)$, so $\sigma$ must fix $M(x ; w)$ pointwise. Thus $M(x ; w)=M(w) \cap$ $\operatorname{dcl}(w \cup Y)$. But $(\operatorname{acl}(x \cup\{p\}): p \in D-x)$ is an independent set over $\operatorname{acl}(x)$ $=M(x)$. Hence $M(w) \perp Y \mid M(x)$, so $M(w) \perp Y \mid(M(x) \cup w)$. Moreover, $\operatorname{tp}(Y / \operatorname{acl}(x) \cup M(w))$ is the unique nonforking extension of $\operatorname{tp}(Y / \operatorname{acl}(x))$, so a fortiori it is the unique (nonforking) extension of $\operatorname{tp}(Y / M(x) \cup w)$. So $\operatorname{tp}(Y / M(x) \cup w) \vdash \operatorname{tp}(Y / M(w))$. By restriction, $\operatorname{tp}(M(x ; w) / M(x) \cup w) \vdash$ $\operatorname{tp}(M(x ; w) / M(w))$. Since $M(x ; w) \subset M(w)$, it follows that $M(x ; w) \subset$ $\operatorname{dcl}(M(x) \cup w)$.

(c) $M(w \cap F)$ is algebraically closed, and $M(w) \perp \operatorname{acl}(F) \mid \operatorname{acl}(w \cap F)$.

(d) We have to show that $[G(r-1 ; w), G(k+1 ; w)] \subseteq G(x, w)$ for each $x \in[D]^{k}$. Fix $x \in[D]^{k}$. Choose $n$ sufficiently large so that $G(x, w, n)=$ $G(x ; w)$ for $w \in[D]^{r}$ with $x \subset w$, and fix $F \supset x$ with $\operatorname{card}(F)=n$. Let $W=\{w: x \subset w \subset F, \operatorname{card}(w)=r\}$. Choose a point $p \in W-x$, and divide $W$ into two sets: $W_{0}=\{w \in W: p \notin w\}, W_{1}=\{w \in W: p \in w\}$.

$\left(*_{1}\right)$ For $w \in W, \operatorname{Aut}\left(M(w) / \bigcup\left\{M\left(w^{\prime}\right): w^{\prime} \in W, w^{\prime} \neq w\right\}\right)=G(x, w)$. This is by the choice of $n$.

$\left(*_{2}\right)$ For $w \in W_{1}, \operatorname{Aut}\left(M(w) / \bigcup\left\{M\left(w^{\prime}\right): w^{\prime} \in W_{1}, w^{\prime} \neq w\right\}\right) \supseteq$ $G(k+1, w)$. In fact the group on the left contains $G(x \cup\{p\}, w)$ explicitly.

$\left(*_{3}\right)$ For $w \in W_{1}, \operatorname{Aut}\left(M(w) / \bigcup\left\{M\left(w^{\prime}\right): w^{\prime} \in W_{0}\right\}\right) \supseteq G(r-1, w)$. This follows from (c), applied to $F-\{p\}$; it shows that the group on the left contains $\operatorname{Aut}(M(w) \mid M(w-\{p\}))$; this in turn contains $G(w-\{p\}, w)$, hence certainly $G(k+1, w)$.

Now pick $w \in W_{1}$ and $\sigma, \tau \in G(k+1, w)$. By $\left(*_{2}\right)$ and $\left(*_{3}\right)$, we can find extension $\bar{\sigma}, \bar{\tau}$ of $\sigma, \tau$ to automorphisms of $\bigcup\left\{M\left(w^{\prime}\right): w^{\prime} \in W\right\}$, such that $\bar{\sigma}$ is the identity on $M\left(w^{\prime}\right)$ for $w^{\prime} \in W_{1}-\{w\}$ and $\bar{\tau}$ is the identity of $M\left(w^{\prime}\right)$ for $w^{\prime} \in W_{0}$. It follows that the commutator $[\bar{\sigma}, \bar{\tau}]$ is the identity on $M\left(w^{\prime}\right)$ for $w^{\prime} \in W-\{w\}$. By $\left(*_{1}\right),[\sigma, \tau]=[\bar{\sigma}, \bar{\tau}] / M(w)$ is in $G(x ; w)$. Since $\sigma, \tau$ were arbitrary, the claim is proved.

Note that $\operatorname{Aut}\left(M_{r, 0} / M_{r, r-1}\right)$ embeds into the product, over all $w \in[D]^{r}$, of $\operatorname{Aut}(M(0 ; w) / M(r-1 ; w))$. This group is isomorphic to

$$
\operatorname{Aut}(M(w) / M(r-1 ; w)) / \operatorname{Aut}(M(w)) / M(0 ; w))=G(r-1 ; w) / G(0 ; w) .
$$

Thus by (d), Aut $\left(M_{r, 0} / M_{r, r-1}\right)$ is nilpotent of class $r$. By Claim (b), $M_{r, r-1}=$ $M_{r-1}$, proving the proposition.

Remark 4.9. It seems possible that "nilpotent" can be replaced by "Abelian". The proof of the preceding proposition showed that the groups $G(\{d\} ; u)$ ) are central in $G(u) / G(0 ; w)$ for $d \in w$. It may be that they generate $G(w)$ over $G(0 ; u)$.

Proposition 4.10. Let $M \in D$. Then $\operatorname{Aut}(M)$ has a sequence of closed subgroups $G_{0} \subset G_{1} \subset \cdots \subset G_{n}$ such that $G_{0}=(1), G_{n}=\operatorname{Aut}(M), G_{i+1}$ is normal in 
$G_{i}$, and the quotient $G_{i+1} / G_{i}$ has one of the following forms (as a topological group).

(i) A finite group.

(ii) $F^{\omega}$, where $F$ is a finite group.

(iii) The full symmetric group on $\omega$.

(iv) $S^{\omega}$, where $S$ is the full symmetric group of $\omega$.

Proof. The totally categorical case is immediate from 4.8. One has a JordanHölder decomposition $(1)=G_{0} \subseteq G_{1} \subseteq \cdots \subseteq G_{r}=\operatorname{Aut}(M)$ in which $G_{r} / G_{r-1}$ is finite, $G_{r-1} / G_{r-2}=\operatorname{Aut}\left(D / M_{0}\right)$ is the full symmetric group on an infinite set $D$, and each subsequent $G_{i+1} / G_{i}$ is either of the form $\operatorname{Aut}\left(N^{*} F / N\right)$ for a structure $N \in \mathbf{D}_{1}$ and a finite $F$, or Abelian.

$$
\operatorname{Aut}\left(N^{*} F / N\right)=\prod\left\{\operatorname{Aut}\left(F(w) / F_{0}(w): w\right)\right\}
$$

is of type (ii). As for the Abelian case, remember that $G_{i+1} / G_{i}$ is profinite of bounded exponent. Being Abelian, it has a further refinement $G_{i+1} \supset G_{i+1}^{\prime} \supset$ $\cdots \supset G_{i}$ in which every factor has prime exponent. By Pontryagin duality, the only infinite separable compact Abelian group of exponent $p$ is $(Z / p Z)^{\omega}$. This falls into case (ii) again. This finishes the totally categorical case. It is not hard to deduce a slightly stronger fact: if $M \in \mathbf{D}_{1}$ and $N$ is any 0-definable subset of $M$, then $\operatorname{Aut}(G / N)$ has a decomposition of the same form.

The general case is done by induction on the essential multiplicity of $M$. Let $D, D_{0}, D_{1}, D_{2}, D_{3}, \hat{D}, E=\bigcup\left\{P_{b}: b \in D_{3}\right\}$ be as in the proof of 3.1. Let $N=\hat{D} \cup E$. We have exact sequences $1 \rightarrow \operatorname{Aut}(M / N) \rightarrow \operatorname{Aut}(M) \rightarrow \operatorname{Aut}(N) \rightarrow$ 1 and $1 \rightarrow \operatorname{Aut}(N / \hat{D}) \rightarrow \operatorname{Aut}(N) \rightarrow \operatorname{Aut}(\hat{D}) \rightarrow 1$, so it suffices to show that $\operatorname{Aut}(M / N), \operatorname{Aut}(N / \hat{D})$ and $\operatorname{Aut}(\hat{D})$ have decomposition sequences of the right form.

$\operatorname{Aut}(M / N)$ : Consider $N$ as a structure in D. By Theorem 3.1 (and the fact that there are no projective spaces around) there exists an expansion $\widetilde{N}$ of $N$ (by some relations $R_{1}, \ldots, R_{k}$ ) such that $\widetilde{N}$ is totally categorical, of disintegrated type. Let $\widetilde{M}$ be $M$ enriched with the relations $R_{1}, \ldots, R_{k}$. Obviously every automorphism of $M$ over $N$ fixes $R_{1}, \ldots, R_{k}$; so $\operatorname{Aut}(M / N)=$ $\operatorname{Aut}(\widetilde{M} / \widetilde{N}) . \widetilde{N}$ is totally categorical, so by the previous paragraph $\operatorname{Aut}(\widetilde{M} / \widetilde{N})$ admits a tower of the required form.

$\operatorname{Aut}(N / \hat{D})=\operatorname{Aut}(E / \hat{D}) \approx \prod\left\{\operatorname{Sym}\left(P_{b}\right): b \in D_{3}\right\}$, so this is a topological group of type (iv).

$\operatorname{Aut}(\hat{D})$ be a decomposition sequence by the induction hypothesis.

Combining the towers gives one for $\operatorname{Aut}(M)$.

We now state the generalizations to $\mathbf{C}_{1}$.

Theorem 4.4C. Let $M \in \mathrm{C}_{1}$, and let $D$ be strictly minimal and 0-definable. Then there exists an expansion by constants $N$ of $M$ such that $N$ has the form $N_{1}{ }^{* 2} F_{2}{ }^{*}{ }^{* n} F_{n}$, where $F_{2}, \ldots, F_{n}$ are finite structures, and $N_{1}$ is a structure 
in $\mathbf{C}_{1}$ of rank 1. Moreover, $N$ may be obtained from $M$ by simply adding $a$ name for a finite subset of $D$.

Theorem 4.8C. Let $M \in \mathbf{C}_{1}$. Then there exist 0-definable subsets of $M^{\mathrm{eq}}$, $M_{1} \subseteq M_{2,0} \subseteq M_{2} \subseteq M_{3,0} \subseteq \cdots \subseteq M_{r, 0} \subseteq M_{r}$, such that:

(a) $\operatorname{dcl}\left(M_{1}\right)=\operatorname{dcl}(\operatorname{acl}(\varnothing) \cup P)$, where $P$ is a modular 0-definable strictly minimal set.

(b) $M_{i}=M_{i, 0}^{* 1} F_{i}$ for some finite structure $F_{i}$.

(c) $\operatorname{Aut}\left(M_{i+1,0} / M_{i}\right)$ is nilpotent.

(d) $r=\operatorname{rk}(M)$, and $M \subseteq M_{r}^{*}$.

Corollary 4.10C. Let $M$ be $\aleph_{0}$-categorical, $\aleph_{0}$-stable of modular type, and let $G=\operatorname{Aut}(M)$. Then $G$ has a topological Jordan-Hölder sequence $(1)=G_{0} \subset$ $G_{1} \subset \cdots \subset G_{n}=G$ such that for each $i, G_{i+1} / G_{i}$ is isomorphic (as a topological group ) to one of the following:

(a) a finite group,

(b) the full symmetric group on $\omega$,

(c) the full projective general linear group on an infinite dimensional projective space over a finite field.

$\left(\mathrm{a}^{\omega}\right),\left(\mathrm{b}^{\omega}\right),\left(\mathrm{c}^{(\omega)}\right)$ : The full product $H^{\omega}$, where $H$ is as in $(\mathrm{a}),(\mathrm{b}),(\mathrm{c})$, respectively.

$4.4 \mathrm{C}$ and $4.10 \mathrm{C}$ are proved similarly to 4.4 and 4.10 . The proof of $4.8 \mathrm{C}$ uses the definition of $M_{i, 0}$ from 4.8 (it is the "linked" part), and the following lemma to prove nilpotency:

Lemma 4.11. Let $G_{1}, \ldots, G_{m}$ be groups, and let $H$ be a subgroup of $G_{1} \times$ $\cdots \times G_{m}$. For $w \subseteq\{1, \ldots, m\}$, let $\pi_{w}$, be the restriction to $H$ of the canonical projection of $G_{1} \times \cdots \times G_{m}$ onto $\prod_{i \in u^{\prime}} G_{i}$. Assume $\pi_{" \prime}$ is 1-1 whenever $\operatorname{card}(w)=m-1$, and $\pi_{w}$ is onto whenever $\operatorname{card}(w)=2$. Then each $G_{i}$ is nilpotent of class $m-2$.

It is possible to find finite groups $G$ and $H \leq G^{4}$ satisfying the hypothesis with $m=4$, and with $G_{i}$ non-Abelian. However, such groups cannot fit as $G(w)$ for $w \in[D]^{3}, D$ a disintegrated Grassmannian. It is unclear whether non-Abelian examples exist in higher dimensions or with less constrained links.

\section{REFERENCES}

[A-Z] G. Ahlbrandt and M. Ziegler, Quasi-finitely axiomatizahle totally categorical theories, Ann. Pure Appl. Logic 30 (1986), 63-82.

[C] G. Cherlin, Quasi-finite axiomatizability' of structures of modular type, preprint.

[CHL] G. Cherlin, L. Harrington and A. Lachlan, $\aleph_{(0)}$-categorical, $\aleph_{(0)}$-stahle structures, Ann. Pure Appl. Logic 28 (1985), 103-135.

[GLR] R. L. Graham, K. Leeb and B. L. Rothschild, Ramsey's theorem for a class of categories, Adv. in Math. 8 (1972), 417-433.

[Hi] G. Higman, Ordering by divisibility in ahstract algehra, Proc. London Math. Soc. (3) 2 (1952), 326-336. 
[Hr] E. Hrushovski, Doctoral dissertation, Univ. of California, Berkeley, 1986.

[L] A. H. Lachlan, Structures coordinatized by indiscernible sets, preprint.

[PH] A. Pillay and E. Hrushovski, Weakly normal groups, ASL Orsay Conf. proceedings, 1985.

[T] S. Thomas, Groups acting on infinite dimensional projective spaces, preprint.

[Z] B. Zilber, Strongly minimal countable categorical theories. II-III, Siberian Math. J. 25 (1984), 396-412, 559-571.

Department of Mathematics, MIT 2-277, Cambridge, Massachusetts 02139 